\title{
Biology and pathogenesis of human osteosarcoma (Review)
}

\author{
JUDSON WELBER VERÍSSIMO DE AZEVEDO ${ }^{1}$, THALES ALLYRIO ARAÚJO DE MEDEIROS FERNANDES ${ }^{2}$, \\ JOSÉ VERÍSSIMO FERNANDES JR ${ }^{3}$, JENNER CHRYSTIAN VERÍSSIMO DE AZEVEDO ${ }^{4}$, \\ DANIEL CARLOS FERREIRA LANZA ${ }^{5}$, CHRISTIANE MEDEIROS BEZERRA ${ }^{6}$, VÂNIA SOUSA ANDRADE ${ }^{6}$, \\ JOSÉLIO MARIA GALVÃO DE ARAÚJO ${ }^{6}$ and JOSÉ VERÍSSIMO FERNANDES ${ }^{6}$ \\ ${ }^{1}$ Orthopedic Trauma Clinic Service, Deoclécio Marques Hospital, 59141-085 Parnamirim, RN; \\ ${ }^{2}$ Department of Biomedical Sciences, University of Rio Grande do Norte State, 59607-360 Mossoró, RN; \\ ${ }^{3}$ Orthopedic Trauma Clinic Service, Getulio Vargas Hospital, 50630-060 Recife, PE; ${ }^{4}$ Department of Pediatrics, University \\ Hospital Onofre Lopes, Federal University of Rio Grande do Norte, 59012-300 Natal, RN; Departments of ${ }^{5}$ Biochemisty, \\ and ${ }^{6}$ Microbiology and Parasitology, Federal University of Rio Grande do Norte, 59072-970 Natal, RN, Brazil
}

Received April 17, 2019; Accepted September 10, 2019

DOI: $10.3892 / 01.2019 .11229$

\begin{abstract}
Osteosarcoma (OS) is a bone tumor of mesenchymal origin, most frequently occurring during the rapid growth phase of long bones, and usually located in the epiphyseal growth plates of the femur or the tibia. Its most common feature is genome disorganization, aneuploidy with chromosomal alterations, deregulation of tumor suppressor genes and of the cell cycle, and an absence of DNA repair. This suggests the involvement of surveillance failures, DNA repair or apoptosis control during osteogenesis, allowing the survival of cells which have undergone alterations during differentiation. Epigenetic events, including DNA methylation, histone modifications, nucleosome remodeling and expression of non-coding RNAs have been identified as possible risk factors for the tumor. It has been reported that p53 target genes or those genes that have their activity modulated by $\mathrm{p} 53$, in addition to other tumor suppressor genes, are silenced in OS-derived cell lines by hypermethylation of their promoters. In osteogenesis, osteoblasts are formed from pluripotent mesenchymal cells, with potential for self-renewal, proliferation and differentiation into various cell types. This involves complex signaling pathways and multiple factors. Any disturbance in this process can cause deregulation of the differentiation and proliferation of these cells, leading to the malignant phenotype. Therefore, the origin of OS seems to be multifactorial, involving the deregulation of differentiation of mesenchymal cells and
\end{abstract}

Correspondence to: Dr Thales Allyrio Araújo de Medeiros Fernandes, Department of Biomedical Sciences, University of Rio Grande do Norte State, Rua Miguel Antônio da Silva Neto, S/N, Aeroporto, 59607-360 Mossoró, RN, Brazil

E-mail: thalesallyrio@uern.br

Key words: bone tumor, osteosarcoma, osteosarcoma biology, osteosarcoma pathogenesis tumor suppressor genes, activation of oncogenes, epigenetic events and the production of cytokines.

\author{
Contents \\ 1. Introduction \\ 2. Biology of human OS \\ 3. Role of differentiation of mesenchymal stem cells \\ 4. Role of DNA changes \\ 5. Role of deregulating the expression of tumor suppressor \\ genes \\ 6. Regulation of oncogene expression \\ 7. Role of epigenetic mechanisms \\ 8. Role of non-coding RNAs \\ 9. Role of cytokines \\ 10. Conclusion
}

\section{Introduction}

Osteosarcoma (OS) is a tumor characterized by the presence of malignant mesenchymal cells produced in the bone stroma (1). The incidence of this tumor in the general population is 2-3 cases/million/year, and it is higher in adolescence, when the annual incidence peaks vary from $8-11 /$ million/year. Among adolescents age group of $10-19$ years, it represents $15 \%$ of all extracranial tumors, being 1.4 times more frequent in men than in women $(2,3)$. A second peak of OS in adults over 65 years of age has been reported, but it is likely to represent a second malignancy, often related to Paget's disease (4). In general, OS is most commonly characterized by an appendicular primary tumor with a high rate of metastasis to the lungs, which usually appears during the first or second decade of life $(5,6)$.

Different studies point to pre-osteoblasts and osteoblasts being the cells which give rise to tumors $(7,8)$. As an important component of the tumor microenvironment, mesenchymal stem cells (MSCs) appear to play an important role in mediating and proliferation in many cancers, including OS. The tumor 
microenvironment exerts different effects on virgin MSCs, in which some cytokines such as SDF-1, MIF, and TGF- $\beta$ recruit these cells to the tumor site, where they are stimulated by the paracrine network and undergo a series of functional transformations. The action of INF- $\gamma$, TNF- $\alpha$ and IL- $1 \alpha$ strengthen the growth-promoting effects of MSCs, while INF- $\gamma$, TNF- $\alpha$ and TGF- $\beta$ enhance the ability of MSCs to promote tumor metastases. In addition, MSCs may differentiate into associated fibroblasts to cancer under the stimulation of TGF- $\beta$ (9).

MSCs first differentiate into chondrocytes during the endochondral bone formation process until adolescence, which generate new cartilage in GP and after are slowly replaced by osteogenic progenitor cells and osteoblasts to produce bone (10). Interestingly, the p53 cellular protein acts as a negative regulator of osteoblastogenesis under normal conditions, repressing transcription factor such as Osterix and Runx2 (11), which are required in the initial osteogenesis phase in osteoprogenitor cells $(12,13)$. However, Runx 2 may act by inhibiting the function of p53 in activating apoptosis by inducing c-MYC transcription by histone modifications (14). This explains the highly elevated Runx 2 expression levels in OS cells. While, Runx 2 may have dual role as a tumor suppressive and as oncoprotein, depending on its cellular levels and context, and its regulation (15).

MSCs represent a source of osteogenic progenitor precursor cells which give rise to osteoblasts. Thus, mutations in the TP53 gene of these cells can lead to defects in controlling cell growth, increasing the risk of developing OS (16).

However, the occurrence of mutations is not the most common event in this type of tumor. Rather, it is best characterized by deregulation of the expression of tumor suppressor genes such as retinoblastoma $(R B 1)$ and TP53, aneuploidy, chromosome structure disruption and uncontrolled cell cycles $(17,18)$. This suggests the possibility of a defect in surveillance or DNA repair mechanisms as one of the possible causes of the tumor's genesis (6).

Epigenetic events are also identified as risk factors for OS, since the DNA methylation pattern of specific genes or gene regions and histone modifications may be involved in tumor development (19). In addition, the methylation levels and silencing of gene encoding tumor suppressor micro-RNAs (miRs) have been described as specific events in human OS cell lines (20). The overexpression of the IGF2 growth factor and of the IRXI gene mediated by the hypomethylation of its promoters has also been reported as an inducer of metastasis in this tumor $(21,22)$.

Bone tissue is highly specialized and has many important signaling pathways to its homeostasis which require crosstalk between bone and immune cells performed by chemical mediators such as cytokines. This is evidenced by the fact that osteoclast formation requires the receptor activator of nuclear factor kappa-B (RANKL) and of macrophage colony-stimulating factor (M-CSF). In turn, RANKL is produced by osteoblast and activated $\mathrm{T}$ cells to regulate osteoclast differentiation, at the same time M-CSF is produced by immune cells and stimulates the expression of RANKL by osteoclast precursor cells such as monocytes and macrophages. In addition, other factors secreted by immune cells may promote or suppress the formation of osteoclasts. This shows the existence of a complex network of communication between cells triggering the immunomodulatory mechanism which may play an important role in tumor development (23).
In this review we present some recent advances on the biology and pathogenesis of OS, with emphasis on the probable mechanisms involved in its initiation and progression. The literature search was conducted using the PubMed (National Institutes of Health; ww.ncbi.nlm.nih.gov/pubmed), Scopus (Elsevier; www.scopus.com/scopus/home.url), and Web of Knowledge (Thomson Reuters; wok.mimas.ac.uk) electronic databases using the following keywords: Osteosarcoma, osteosarcoma biology, osteosarcoma pathogenesis, osteosarcoma signaling pathways, osteosarcoma genetics, osteosarcoma epigenetic, and cytokine profile in osteosarcoma. Several hundred articles were found in the surveyed databases, however only those which were considered most relevant which had been published in impact journals and were conducted by groups with recognized knowledge in the area were selected.

\section{Biology of human OS}

OS is a tumor that is most frequent in the rapid growth phase of the long bones which occurs during puberty. More than $50 \%$ of the cases have origin in the epiphyseal GP of the distal femur and proximal tibia where the bone growth develops, being responsible for much of the height increase which occurs during adolescence (4).

Among the possible mechanisms that contribute to OS development are alterations in the differentiation pathway of MSCs in mature osteoblasts (24). Furthermore, abnormal expression of oncogenes and of tumor suppressor genes triggered by genetic and epigenetic events lead to deregulation of important cell signaling pathways, thereby creating a favorable environment to malignant transformation $(25,26)$. This is because there is a greater bone turnover during the growth phase, and so the possibility for defects to occur in the differentiation process and in the signaling pathways is amplified (4).

MSCs of the bone marrow stroma are undifferentiated cells with potential for self-renewal, proliferation, and differentiation for bone, muscle, tendon, and fat formation (27). Many endogenous and exogenous factors are involved in the osteogenesis process to form osteoblasts by the osteogenic pathway which leads to the differentiation of MSCs into osteoblasts. Deregulation of these factors, or exposure to new non-native stimuli such as pro-inflammatory cytokines and pro-tumor, may cause an imbalance between cell differentiation and proliferation, contributing to a malignant phenotype $(28,29)$. As main component of the tumor microenvironment, MSCs can mediate cellular proliferation and metastasis, as well as drug resistance in OS (9).

Current knowledge indicates that OS exhibit a wide range of genetic, epigenetic, and molecular changes, including gains, losses, or arrangements of chromosomal regions; inactivation of tumor suppressor genes; and deregulation of cell signaling pathways (17). Each of the mechanisms mentioned above will be presented with more detail in the following sections.

\section{Role of differentiation of mesenchymal stem cells}

The main function of MSCs is self-renewal which requires a multi or pluripotency state, remaining undifferentiated and proliferative to maintain homeostasis during the development phase or even throughout life in order to maintain body 
homeostasis or make repairs. Such properties are in many ways analogous to those of cancer cells, since the unlimited potential for proliferation, also known as immortality, is the most striking feature of malignant tumors (30). In addition, stemness maintenance is achieved by restricting the differentiation, apoptosis, and cellular senescence, which are also characteristic of transformed cells (31).

MSCs are present in many human organs and comprise a heterogeneous population of self-renewing cells, and their morphology, immunophenotype, and differentiation potential depend on their tissue origin. Specific populations of the stroma maintain the regeneration process of the tissue where they reside, but some of them have much greater plasticity and differ across multiple cell lineages. Thus, MSCs not only contribute to the structural repair of tissues, but also possess strong immunomodulatory and anti-inflammatory properties and can influence tissue repair through modulation of the local environment (32).

In a parallel, functional and phenotypic analyses of normal MSCs and MSCs derived from OS were performed to evaluate the pre-malignant stages of the tumor in a murine MSC system in which tumor development was demonstrated after grafting of transformed MSCs. This is substantial evidence to support the hypothesis that this tumor originates from MSCs. Analysis of different passages of MSCs using COBRA-FISH karyotyping and $\mathrm{CGH}$ array revealed the occurrence of aneuploidization, translocations, and homozygous loss of $C D K N 2$ region of the genome these cells, in which encoding cyclin-2A dependent kinase inhibitor is a mediator of malignant transformation of MSCs. Interestingly, the expression of the CDKN2 gene product, the p16 protein, was reduced in the samples of 88 patients with OS, confirming the results obtained by the murine system (33).

In another study was found that that the SOX5 gene, which encodes a family of transcription factors involved in regulating embryonic development and which determines the destination of cells, is significantly expressed in OS tissue and in cell line-derived tumor. In addition, the expression of SOX 5 promoted epithelial-mesenchymal transition (EMT) and increased migration and invasion of tumor cells (34).

A recent study involving crosstalk between OS cells and MSCs, mediated by extracellular vesicles (EVs) which play an important role in initiating and progressing cancer, showed strong evidence of MSCs participating in the origin of OS. MSCs and pre-osteoblasts were treated with OS-EVs at different times, and their epigenetic signature was evaluated through of methylation analysis of LINE-1 (long interleaved element) and tumor suppressor genes. This shows that OS-EVs mediate LINE-1 hypomethylation in MSCs and LINE-1 hyper methylation in the pre-osteoblasts, indicating that MSCs, but not pre-osteoblasts, are susceptible to epigenetic transformation. Thus, OS-EVs modulate the fate of MSCs, regulating epigenetic status and influencing gene expression related to bone microenvironment remodeling. This suggests that epigenetic regulation appears to be an early event in transforming MSCs during OS development (35).

\section{Role of DNA changes}

The TP53 gene plays a critical role in the regulation of both the cell cycle and apoptosis, and its product (the p53 protein) is synthesized in response to stress situations due to tensions such as DNA damage, hypoxia, and oncogene activation. This gene frequently undergoes negative selection during tumorigenic transformation. Mutations in the TP53 gene in response to DNA damage can promote uncontrolled cell cycles, inhibit senescence and cell death by apoptosis, thereby increasing the genomic instability. This leads to an accumulation of mutations and cell survival, in turn increasing the risk of malignant transformation, including OS development (36).

The occurrence of mutation in OS was investigated in a study in which the whole-exome and RNA-sequencing of 59 tumor/normal pairs of samples revealed that only the TP53 tumor suppressor gene showed mutation with significant frequency in all the samples. The mean non-silent somatic mutation rate was 1.2 mutations per mega base with a median of 230 somatic rearrangements per tumor. There was great genetic intratumor heterogeneity, with the presence of complex chains of rearrangements and hypermutation in almost all cases $(37,38)$.

Tumor analysis by multiregional whole-exome and whole-genome sequencing in 86 tumor regions from 10 patients with OS revealed an evolutionary genomic disparity between primary OS and its pulmonary metastases, where the metastases exhibited a higher mutational load and genomic instability compared to the primary tumor. The mutated genes were enriched in the PI3K-Akt pathway at both the early and late stages of tumor evolution and in the MAPK pathway in the metastatic stage. However, metastases showed improved immunogenicity, including increased neoantigen loading, and also improved PD-L1 expression, an immunoglobulin superfamily gene, and having more infiltrating lymphocytes compared to the primary tumor. This suggests that metastases should be treated separately from their original tumors by means of personalized metastasis therapy, which requires real-time genetic analysis after pulmonary metastasis (39).

Silent mutations in the TP53 and/or RB1 genes have been reported to be the leading cause of sporadic development of OS (11). In vitro and in vivo study comparing MSCs with OS malignant cells directed to the TP53 and RBI using transgenic mice with these silenced genes in its MSCs showed that by only excluding TP53, the OS incidence could reach $60 \%$ of cases (40). It has been shown that p53 act as a guardian of the osteogenic differentiation of MSCs into myogenic, adipogenic, hematopoietic and neural adult cells (11).

Different studies point the pre-osteoblasts and osteoblasts as cells which give rise to OS $(8,41)$, suggesting that the cellular microenvironment is critical in determining the fate of MSCs in tumor formation (10). The osteogenic differentiation of MSCs with defective or mutant p53 may affect signaling and its microenvironment, and possibly contribute to tumor initiation (11).

As MSCs represent the source of osteogenic progenitor cells and osteoblasts, thus mutations in TP53 gene of these cells play a decisive role in proliferation, compromising the maturation, negatively regulating their differentiation, and interfering with cell processes such as ontogenesis (11). This is due to the reduced expression of key genes encoding transcription factors involved in the early stages of osteogenic differentiation, including Runx2 and Osterix $(14,42)$. Under 
normal conditions, Runx2 and Osterix expression is strictly regulated during osteogenic differentiation of progenitor cells into osteoblasts and osteocytes, ensuring balanced bone remodeling. In vitro silencing of the TP53 gene in embryonic mouse fibroblasts induces increased Osterix and Runx2 expression levels in MSCs. This compromises the differentiation of osteoblasts in mature osteocytes, causing damage to bone remodeling, resulting in the osteosclerotic phenotype observed in p53-deficient mice $(11,42)$.

It has been demonstrated that p53 not only regulates the genomic stability of MSCs, but also regulates the cell differentiation program including osteogenesis and bone remodeling to prevent the onset of bone tumor. The absence of the p53 function in the regulation of the differentiation of MSCs in osteoblasts due to mutational events or silencing can start the tumor as a result of changes in osteogenesis, bone homeostasis, and bone remodeling $(11,40)$.

OS is a heterogeneous tumor containing cells at various stages of differentiation during ontogenesis (7). Thus, it was proposed that mutation in TP53 gene may affect osteogenic differentiation of MSCs and significantly contribute to tumor onset by the following mechanisms: (1) Stop acting as a transcription factor, suppressing multipotent progenitor cell differentiation to mediate early osteogenic differentiation (2) promoting genomic instability and uncontrolled proliferation of MSCs; and (3) deregulating the immune activity of MSCs, increasing the secretion of growth factors and chemokines. This suggests an important role for $\mathrm{p} 53$ function defects in OS development. Evidence of this is that the osteosclerotic condition imposes the OS phenotype in p53-deficient mice (11).

It has been reported that frizzled-related secreted protein 2 (SFRP2) has an oncogenic role in OS development associated with TP53 gene mutation, and that the high expression of this protein correlates with poor prognosis of OS patients (43). Thus, induced pluripotent stem cells (iPSCs) obtained from patients with Li-Fraumeni syndrome that have mutations of the TP53 germline were used to analyze the role of SFRP2 in OS. It has been found that ectopic SFRP2 overexpression in normal osteoblast precursors containing TP53 gene mutation is enough to suppress normal osteoblast differentiation and promote OS phenotypes by inducing oncogenic molecules such as FOXM1 and CYR61, independently of $\beta$-catenin. On the other hand, inhibition of SFRP2, FOXM1 or CYR61 suppresses the tumorigenic process. This demonstrates that the oncogenic role of SFRP2 in OS development is due to its ability to induce oncogenic molecules such as FOXM1 and CYR61 in the presence of mutations in the TP53 gene (44).

A dominant subclone was identified in samples from patients with successive recurrences after sequencing the exome and germ cell DNA from cells collected from a patient with chemoresistent and metastatic OS over 3 years at 3 different times and after comparing allele frequencies of the different samples. This clone presented two remarkable features, consisting in a novel translocation in TP53-KPNA3 allele and the loss of the wild-type TP53 allele. Lastly, a meta-analysis study which included 8 publications covering 210 patients with OS evaluated the effect of the mutations in TP53 gene and concluded that mutations in this gene were associated with smaller 2-year overall survival of patients. The data show that mutations in TP53 gene have one unfavorable impact on the 2-year overall survival when compared to the wild type (45).

\section{Role of deregulating the expression of tumor suppressor genes}

Among the tumor suppressor genes includes WWOX, whose function is suppressed or attenuated in most human tumors. In a Wwox-deficient mice model it was demonstrated that these animals developed OS and a bone metabolic disorder characterized by hypocalcemia and osteopenia. In addition, deletion of the WWOX gene was found in $30 \%$ of OS and the protein product of this gene was absent or reduced in about $60 \%$ of the tumors. It has been found that the tumor suppressor function of $W W O X$ is exerted through its binding to $R U N X 2$, suppressing its transcriptional activity in osteoblasts and tumor cells. Thus, the negative regulation of $W W O X$ results in the maintenance of the RUNX2 activity, creating a conducive environment to the development of OS since low levels of WWOX expression increase proliferation, migration, and invasion of tumor cells $(46,47)$.

The $R B 1$ gene encoding the retinoblastoma protein $(\mathrm{pRB})$ plays a critical role in regulating the transition from the G1 phase to the S phase of the cell cycle. In the absence of mitogenic stimuli, pRB remains hypophosphorylated and bound to E2F, a transcription factor, which prevents the $\mathrm{pRB}$ action on the cell cycle progression, leading to a cycle stop in G1 (48). This function is reversed by phosphorylation of pRB by the cyclin-dependent kinase 4 (CDK4) during normal mitosis, which results in the release of E2F, leading to cell cycle progression. The absence of cell cycle arrest in G1 due to mutations or RB1 silencing removes this cell cycle control point, preventing the repair of DNA damage, and causing genomic instability (49).

The $C D K N 2 A$ gene codes two products through alternative splicing which are functionally and structurally distinct (p16INK4a and p14ARF). The p16INK4a is a negative regulator of $C D K 4$ and therefore the gift of its function leads to an increase in CDK4 expression, which results in inactivating the pRB function in cell cycle arrest (50). Thus, mutations or silencing of the $C D K N 2 A$ gene may lead to inactivation of the pRB function. Curiously, control losses in the cell cycle caused by the loss of $\mathrm{pRB}$ function are reported in most OS cases (18). The p14ARF protein normally acts by removing the ubiquitin E3 MDM2 ligase from the nucleolus, preventing its degradation action of p53 (51). Since p14ARF is expressed from the same locus of $C D K N 2 A$ encoding p16INK4a, its function in pathway p53 is like that of p16INK4a in the pRB pathway, disrupting the cell cycle. Thus, mutations or silencing by methylation of the $C D K N 2 A$ gene also alter p14ARF function and have repercussions on the p53 pathway, promoting cell cycle dysregulation, leading to genomic instability. Mutations affecting p53 function have been described in most OS cases (Fig. 1) $(18,49)$.

A recent study showed that functional genetic single-nucleotide polymorphisms in the CDKN2 gene, locus $\mathrm{A}$ and $\mathrm{B}$ (CDKN2A/B) predict susceptibility to and prognosis of OS in Chinese individuals. The GA and AA genotypes of rs 3217992 in CDKN2A/B are related to increased risk of tumor, and the GA and AA genotypes of rs 3217992 in CDKN2A correlate with higher stage and higher risk of pulmonary metastasis and poor prognosis (52). 


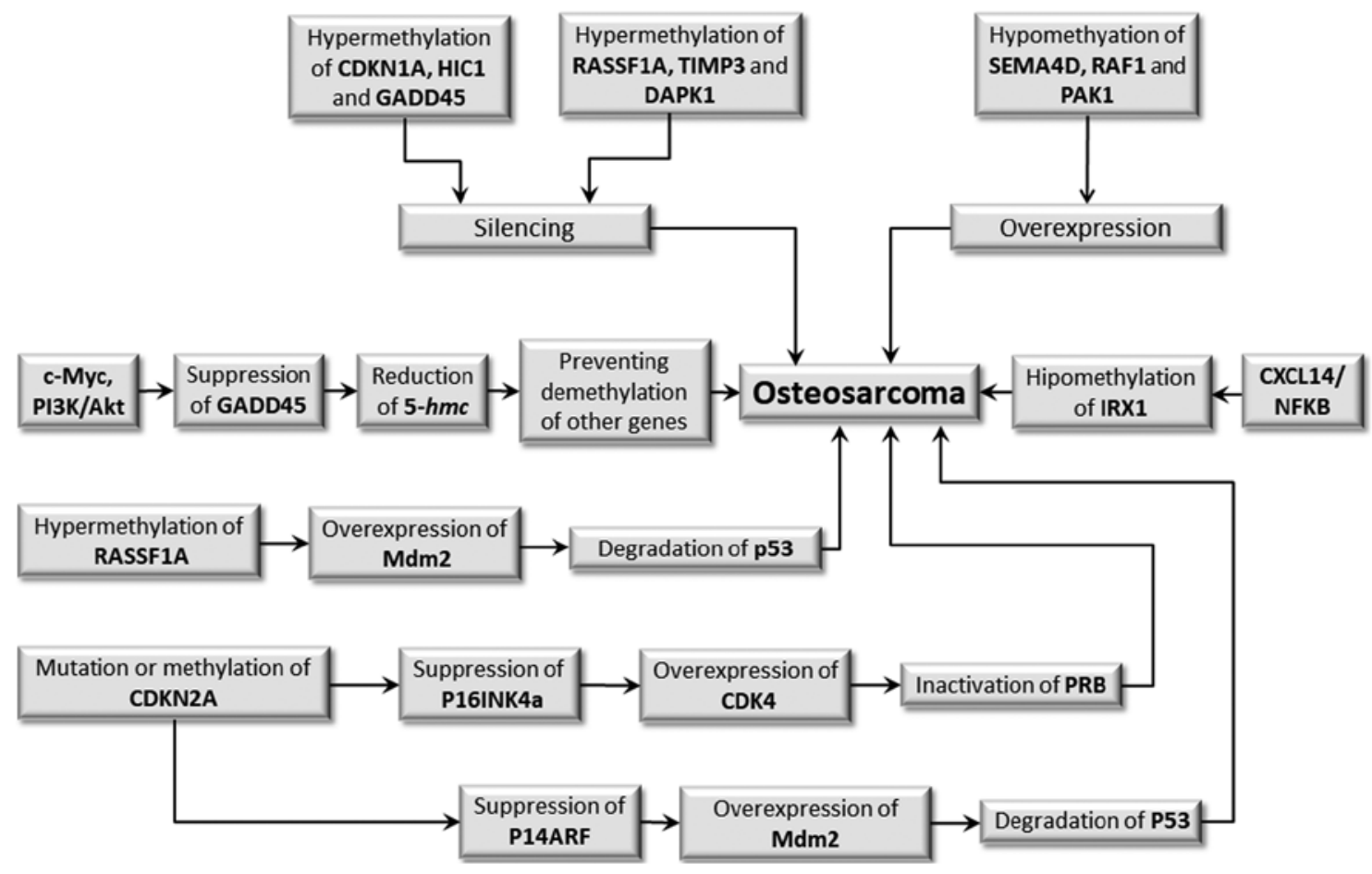

Figure 1. Epigenetic events which may contribute to the initiation and progression of OS. Target genes or those which have p53-modulated activities, including GDKN1A, HIC, GADD45, RASSF1A, TIMP3 and DAPK1, are silenced when hypermethylated. An inverse situation occurs with SEMA4D, RAF1 and PAK1 genes when they are hypomethylated which results in overexpression. However, both conditions may equally favor the development of OS. Silencing by hypermethylation of the RASSF1A gene leads to overexpression of the MDM2 gene, whose product promotes p53 degradation, which in turn results in uncontrolled cell cycle and absence of DNA repair and apoptosis inhibition. The hypomethylation of the IRX1 gene promotes activation of the CXCL14/NFkB signaling pathway, whereas the activation of the cMyc and PI3/Akt signaling pathways results in suppression of the GADD45 gene encoding the 5-hmC production, impeding the demethylation of other genes, which also favors tumor development. Mutation or methylation of the CDKN2A gene reduces the production of p16INK4a, which leads to overexpression of CDK4 and inactivation of pRB. It can also reduce the production of p14INK4a, which in turn suppresses p14ARF, resulting in high Mdm2 levels which degrades p53. Both mechanisms result in an uncontrolled cell cycle, lack of DNA repair and apoptosis inhibition, favoring tumor initiation and progression. OS, osteosarcoma.

\section{Regulation of oncogene expression}

Like what occurs with other tumors, the abnormal activity of oncogenes and tumor suppressor genes is described as a key molecular event underlying the development of OS (25). The $M D M 2$ oncogene whose activity may be dependent or independent of p53 presented frequently increased expression levels in a variety of human tumors, since it significantly impacts the p53 functions and consequently tumorigenesis (51). The product of this gene (the Mdm2 protein) is one of the major negative regulators of p53 as it performs E3 ubiquitin ligase activity which promotes inactivation of p53 function by its degradation. Under normal conditions, Mdm2 suppresses p53 activity to allow cell cycle continuity, but under stress conditions it remains active and promotes cell cycle arrest for correcting possible damage to DNA (53). Overexpression or amplification of the MDM2 locus is detected in OS (54).

The $c-M y c$ oncogene stands out as one of the most studied and whose role in OS pathogenesis is best understood. Moreover, it is found to be overexpressed in more than $10 \%$ of cases of the disease, being correlated with increased recurrence and tumor invasiveness due to the activation of the MEK-ERK pathway, leading to the reduction of apoptotic potential in tumor cells (55). Studies show that inhibition of c-Myc activity results in decreased proliferation, invasion, and viability of the tumor cells $(55,56)$.
The c-Myc oncogene is significantly overregulated in metastatic OS samples and the high expression of this oncogene is associated with poor survival of OS patients. Treatment of OS cells with super enhancer inhibitors THZ1 and JQ1 effectively suppresses OS cell proliferation, migration and invasion (57).

On the other hand, c-Fos is another oncogene which has been found to upregulate in OS cells, being related to a higher rate of tumor metastasis (26). The expression of c-Fos and of Wnt2, a protein implicated in cell oncogenesis and its Fzd9 receptor, was evaluated in OS tissue, tumor-derived MG63 cells and in osteoblasts to investigate the role of c-Fos in OS. MG63 cells were treated with small interference RNA to knockdown c-Fos, aiming to confirm the relationship of c-Fos with Wnt2/Fzd9. The expression of c-Fos, Wnt2 and Fzd9 was found to be markedly higher in OS tissues than in adjacent non-cancerous tissues, and their expression in MG63 was markedly increased compared to osteoblasts. c-Fos knockdown inhibited MG63 cell proliferation, migration and invasion, and promoted MG63 cell apoptosis. In addition, c-Fos knockdown inhibited mRNA and protein expression of Wnt2 and Fzd9. This indicates that the c-Fos action in OS development is done by activating expression of Wnt2 and its Fzd9 receptor (58).

TRIM14 is upregulated in OS samples and cell lines derived from this tumor. Overexpression of TRIM14 increases tumor cell proliferation, cell cycle progression, 
migration and invasion in vitro and promotes tumor growth in vivo. Moreover, TRIM14 overexpression is correlated with tumor progression and low patient survival time. On the other hand, silencing TRIM14 has the opposite effects. In addition, TRIM14 overexpression induced activation of the AKT pathway, while inhibition of AKT expression reversed TRIM14-mediated effects on cell growth and mobility, as well as epithelial-mesenchymal transition. This indicates that TRIM14 functions as an oncogene in OS, positively regulating the AKT signaling pathway (59).

\section{Role of epigenetic mechanisms}

Epigenetics comprises a set of biological phenomena triggered by environmental factors which promote gene expression regulation at the transcription level through chemical modifications in the DNA, such as changes in the pattern of methylation, acetylation, phosphorylation, stable chromatin modifications, and of histones, in addition to nucleosome remodeling and Non-coding RNAs $(60,61)$. These epigenetic modifications occur in key oncogenes, tumor suppressor genes, and transcription factors, leading to cancer initiation and progression (61). Such events may result in alterations in the expression or silencing of genes and of miRs which result in the phenotypic change of the individual without alterations in the DNA sequence (62). Such events are crucial for the development and normal differentiation of different cell lines in the adult individual (63). Unlike the genetic alterations which are irreversible, the epigenetic changes are reversible, allowing us to intervene to reverse the malignant characteristic of a population of cells, returning it to its normal status (19). These chemical modifications in DNA are constantly made and undone throughout the life of the individual, since he or she often encounters agents which promote these phenomena throughout their lives (6). Like what occurs with other types of human cancers, the initiation and progression of OS can be triggered by genetic and epigenetic events that alter the behavior pattern of cells, altering gene expression and/or signaling pathways, which can contribute to malignant transformation (19). Although it exhibits a wide range of genetic and molecular changes, including gains, losses, or rearrangements of chromosomal regions, the more recent knowledge suggests that OS is a disease caused by epigenetic alterations which interrupt the osteoblastic differentiation of MSCs (17). Epigenetic studies have shown extensive reprogramming of each component of the epigenetic machinery of the tumor cells including DNA methylation, histone modifications, nucleosome positioning, and miRs expression (19).

As with other human cancers, OS appears to contain many of these epigenome changes compared to normal osteoblasts, the presumed target cell for transformation. Some studies have analyzed the epigenome-like shape of this tumor, albeit with a low number of cases $(64,65)$. The results confirm the existence of specific heterogeneous methylation events among the different types of human OS and that these differences may help explain the differences in the clinical behavior of subtypes of this tumor (66).

It has recently been observed that the crosstalk between MSCs and extracellular vesicle-mediated OS cells can influence the epigenetic signature of cells through the methylation of transposable elements such as LINE-1 and tumor suppressor genes, modulating the fate of mesenchymal stem cells and the epigenetic status of these cells by altering gene expression related to bone turnover (35). Global changes in epigenetic patterns are a hallmark of cancer, as disturbances in epigenetic processes can lead to altered genetic function and malignant transformation of the cell. The initiation and progression of cancer, traditionally considered as a genetic disease, is now understood as a complex process involving epigenetic abnormalities along with genetic alterations. A better understanding of epigenetic mechanisms in the development of cancer and the role of some of these components in relation to OS is discussed below.

DNA methylation. DNA methylation is a chemical change involving the addition of a methyl group to the cytosine DNA nucleotides which typically occurs in $\mathrm{CpG}$ dinucleotides not randomly distributed in the genome that represents an important epigenetic mechanism used for the prolonged silencing of gene expression (67). The human genome contains long stretches of $\mathrm{CpG}$ islands, with unusually elevated levels of $\mathrm{CpG}$ dinucleotides, concentrated in the promoters of the genes. In general, the $\mathrm{CpG}$ islands of normal gene promoters are not methylated and they are normally expressed (68).

The methylation pattern of a given mammal is established during its development and is normally maintained throughout life, being regulated by the enzymes DNA methyltransferase (DNMT) and demethylase. Changes in the expected pattern of methylation by either hypomethylation or hypermethylation can lead to genomic instability and trigger tumorigenesis (69).

There are three classes of DNMTs in eukaryotes (Dnmt1, Dnmt2, Dnmt3a/Dnmt3b) (70). DNMT1 is the most important and responsible for maintaining DNA methylation levels, while DNMT3a/DNMT3b is involved in methylation again, being responsible for establishing DNA methylation patterns during embryogenesis and setting up genomic imprints during germ cell development (71). Although DNMT2 is not currently considered to be a DNA methylase, this enzyme methylates small transfer RNAs (tRNAs) (72).

Changes in the expected pattern of methylation are it for hypomethylation or hypermethylation can lead to genomic instability and trigger tumorigenesis or maintain the malignant state of cancer cells. Hypermethylation of the promoter of a gene is responsible for its transcriptional inactivation, a common event in cancers. The silencing or activation of genes mediated by aberrant DNA methylation, can affect almost all cell signaling pathways, including those of DNA repair, cell cycle regulation, promotion of apoptosis or control of signaling networks relevant to tumor development (73).

Methylation as the consequent silencing of tumor suppressor genes has been reported in OS. Although $R B$ and TP53 genes are not frequent targets of silencing by methylation, changes in pRB and p53 pathways have been pointed as pathogenic methylation targets, specifically the CDKN2A locus, which encodes the cyclin-dependent kinase inhibitor, p16INK4a, and the inhibitor of Mdm2, p14ARF (7). Several gene targets of p53, or which have its p53-modulated activity, have been found in the methylated form and silenced on OS or xenograft cell lines, including CDKNIA, HICl and $G A D D 45$ (74). In addition, many other tumor suppressor genes are silenced by hypermethylation of their promoter in 
OS-derived cell lines, including RASSF1A, TIMP3, DAPK1, and others (Fig. 1) (6).

It was found that GADD45 gene encoding proteins of the 5-hydroxymethylcytosine $(5 \mathrm{hmC})$ family, which mediates the methylation in osteogenic differentiation, is co-operatively repressed by the c-Myc and PI3K/Akt pathways in OS cells (75). The repression of GADD45 may be due to the aberrant methylation pattern of its promoters. Interestingly, p53 reduce methylation of promoters of tumor suppressor genes, among them RASSF1A (6). However, there are cancer cells that have wild-type TP53, which is down-regulated by means of a p53-RASSF1A-Mdm2 feedback loop which results in hypermethylation of RASSF1A, leading to $M D M 2$ expression which remains bound to $\mathrm{p} 53$, promoting its degradation (76). On the other hand, RASSF1A promotes Mdm2 degradation in a p53-dependent manner, preventing degradation of p53 by Mdm2. The silencing of RASSF1A due to DNA methylation is the explanation for the fact that there are cancer cells which have wild TP53 (77).

A study of gene expression associated with metastasis in OS identified the IRXI gene as a candidate to be gene pro-metastatic when it undergoes little methylation. IRXI encodes a member of the iroquois homeobox protein 1 family, a transcription factor which plays a crucial role in embryonic development and was previously pointed out as a potential tumor suppressor in gastric cancer (78). It was hypothesized that the hypomethylation of IRXI gene promotes pulmonary metastasis of the OS, since overexpression of this gene was strongly associated with the hypomethylation of its promoter in both OS-derived cell lines and in clinical samples obtained from the tumor. These pro-metastatic effects of IRX1 are due to its role as positive regulator of the CXCL14/NF-kB cell signaling pathway. In addition, it has been shown that IRX1 can increase tumor cell metastatic activity both in vitro and in vivo, favoring migration and invasion, as well as promoting resistance to anoikis in the murine model (Fig. 1) (22).

The degree of methylation of more than 1.1 million loci was tested on biopsy samples obtained from patients with OS and analyzed in function of relapse or not of the disease. It was found that patients who had tumor recurrence were more methylated in more than $17 \%$ of the samples, whereas less than $1 \%$ of patients who did not have relapse had high methylation. Moreover, hypermethylation was found in genetic bodies, intragenic regions, and promoters in patients with recurrent disease. It was demonstrated that in $6.6 \%$ of the patients who had relapsed, the promoters of the candidate gene were hypermethylated and $2 \%$ were hypomethylated. A locus at the TLR4 gene demonstrates one of strongest positive associations between DNA methylation and 5 y event-free survival (66). Several candidate oncogenes including SEMA4D, RAFI and $P A K 1$ are also hypomethylated and overexpressed in human OS compared to normal osteoblasts (79). Furthermore, some of these epigenetic changes, including repression or aberrant activation, are associated with loss of expression control at specific loci in OS cells (Fig. 1) (66,79).

A comparative study of the DNA methylation degree of normal samples with those obtained from OS revealed that the promoters of some genes are differentially methylated in the tumor. The pathways and functions affected by these genes were identified through protein-protein interaction (PPI), followed by the identification of genes associated with cancer which had their promoters differentially methylated, wherein 1379 hypermethylated regions and 169 hypomethylated regions were identified. Differential hypomethylation was significantly greater in the toll receptor signaling pathway. In the PPI network, the MAXI interactor signals transducer 1 (MXII), the transcription activator STAT3 and the T-cell acute lymphocytic leukemia 1 (TAL1) had the highest degree of hypermethylation. These genes were identified as being associated with cancer and were hypermethylated in OS cells (80).

The HOTAIR gene has been shown to be highly expressed in OS cells, while knockdown of this gene results in down regulation of DNMT1 with a reduction in overall DNA methylation level. It was further seen that the HOTAIR product represses CDKN2A expression by inhibiting CDKN2A promoter activity by DNA hypermethylation. Mechanistically, HOTAIR acts in OS by suppressing miR-126 expression, which is the negative regulator of DNMT1. Thus, DNMT1 occurs in the absence of miR-126 overexpression, leading to silencing of CDKN2A due to hypermethylation of DNA its promoter, thus favoring tumor development (81).

A methylation status analysis of the whole genome of 19 different OS-derived cell lines and of 6 normal controls was performed and the comparison between the two cell types was established. The differentially methylated sites in tumor cells were analyzed with the $\mathrm{CpG}$ assoc package and a total of 75 sites were methylated in transcription factor binding regions to which 83 transcription factors can bind, which may lead to alteration in the expression of 75 genes being differentially expressed in tumor cells. In addition, several differentially methylated sites have been associated with up-regulation of genes such as SEZ6L2, KIRREL, CEP72 and CDK4, which may play an important role in OS pathogenesis (82).

The hypermethylation of DNA from two $\mathrm{CpG}$ islands adjacent to miR-449c genomic locus results in inhibition of its expression, and consequently abolishes the function of miR-449c as a negative regulator of c-Myc oncogene expression. In this condition, c-Myc passes to be overexpressed, leading to activation of downstream targets, contributing to OS tumorigenesis (83). Analysis of over 11,000 genes for differential methylation level and over 3,000 genes for differential expression in the OS revealed that the functions of genes related to this tumor were mainly enriched in biological processes related to inflammatory/immune response, Pertussis pathways and hematopoietic cell lineage pathways. UBS and NRF were found to be regulated by multiple genes in the OS. Kaplan Meier analysis of genes to OS-associated identified BHMT2, DOCK2, DNALI1 and RIPK3 as significant survival-related genes. SEMA3A and PRAME are included in the 40 genes and within the top 10 of the most differentially expressed genes in OS (84).

Histone modification. Covalent modifications of the amino termini of the histones in nucleosomes play a critical role in the regulation of gene expression (85). Such modifications are even more complex than DNA methylation because they include acetylation, methylation, phosphorylation, ubiquitination, and sumoylation (86). The amino-terminal modifications of these proteins affect the affinities of the chromatin-associated proteins and influence regulation of the dynamic transitions 
between transcriptionally active or silent chromatin states. Thus, the normal state of acetylation of histones and other transcription factors bound to the promoter determines the dynamic equilibrium that is regulated by acetyltransferase and histone deacetylase (HDAC) enzymes. Aberrant acetylation with histone modifications are implicated in anomalous expression of oncogenes and tumor suppressor genes, which ultimately leads to tumorigenesis (87).

Unlike the dynamic equilibrium of acetylation observed in normal cells, histones are typically hypoacetylated in tumor cells (88). Histone methylation may activate or inactivate gene transcription, depending on where methylation occurs. Generally, H3K4, H3K36 and H3K79 methylations are related to active gene transcription, while methylations of $\mathrm{H} 3 \mathrm{~K} 9$, H3K 27 and H4K20 are associated to gene silencing. Thus, modifications of histones interact with DNA methylation and the combined action of the two mechanisms plays a key role in gene expression (89).

WNT5A is a family of genes which encode signaling glycoprotein and its altered expression is associated with various types of cancer. Expression of promoters A and B of the WNT5A gene was studied in normal human osteoblasts, in two SaOS-2 and U2OS OS cell lines, and in tumor tissue. It has been found that both promoters $\mathrm{A}$ and $\mathrm{B}$ are active in normal osteoblasts, being that promoter $\mathrm{B}$ was nearly 11 times more active than promoter $\mathrm{A}$. Three regions enriched with $\mathrm{CpG}$ islands of exon $1 \beta$ of promoter $B$ are highly methylated in both SaOS-2 and U2OS cells. Histone modifications were examined for their involvement in the activity of promoters A and B. It was found that $\mathrm{H} 3 \mathrm{~K} 4 \mathrm{me} 3$, a marker of histone activation, showed a high level of histone modifications in promoter $\mathrm{A}$ and a reduced level of promoter B modifications in cell U20S, suggesting that $\mathrm{H} 3 \mathrm{~K} 4 \mathrm{me} 3$ plays a repressive role, reducing the activity of the promoter B. It has also been found that promoter B is less enriched with the active $\mathrm{H} 3 \mathrm{~K} 4 \mathrm{me} 3$ compared to promoter $\mathrm{A}$ in U2OS and SaOS-2 cells. In addition, there is increased enrichment of the repressive $\mathrm{H} 3 \mathrm{~K} 27 \mathrm{me} 3$ in the promoter $\mathrm{B}$ in SaOS-2 cells. Inhibition of promoter B of the WNT5A gene appears to be an OS characteristic and involves both DNA methylation and histone modifications. These results indicate that histone modifications in the WNT5A gene promoter B reduce the transcription activity of this gene in OS cells (90).

Histone demethylases KDM6A and KDM6B, associated with the demethylation of histone $\mathrm{H} 3$ lysine trimethylation (H3K27me3) were found to be upregulated in OS cells after treatment with cisplatin. Cisplatin-resistant tumors had lower levels of $\mathrm{H} 3 \mathrm{~K} 27 \mathrm{me} 3$ than sensitive OS specimens. In vitro inhibition of histone methyltransferase EZH2 in OS cells decreased H3K27me3 levels and led to cisplatin resistance. On the other hand, inhibition KDM6A and KDM6B demethylases increased $\mathrm{H} 3 \mathrm{~K} 27 \mathrm{me} 3$ levels and reversed cisplatin resistance in vitro and in vivo. This indicates that $\mathrm{H} 3 \mathrm{~K} 27 \mathrm{me} 3$ acts in reducing KDM6A and KDM6B expression by increasing tumor cell sensitivity to cisplatin (91).

Nucleosome remodeling. The conformational changes and changes in position of the nucleosomes along the DNA strand alter the interactions between DNA and histones and interfere in the affinities of transcription factors to DNA (92). Thus, aberrant nucleosome remodeling can cause great damage to the correct functioning of the cell. Remodeling of the nucleosomes has a critical role in the process of normal differentiation and is controlled by ATP-dependent chromatin-remodeling complexes (CRCs). Such complexes act by regulating a wide range of cellular processes, including transcription regulation in response to DNA damage, DNA replication, and determination of cellular identity. In this way, the deregulation of any of these processes can contribute to the cellular transformation and tumorigenesis (90).

It has been shown that both DNA methylation and histone modification as nucleosome remodeling may contribute to transcriptional suppression and gene silencing in human OS. When the $\mathrm{CpG}$ island of the promoter is methylated, the methyl-CpG binding domain proteins (MBDs) will bind to this site instead of the transcriptional activator complex. MBDs will recruit histone deacetylase (HDAC), and consequently the histones are deacetylated. Histone deacetylation increases the overall positive charge of histone tails, which is associated with a more compact heterochromatin structure, causing condensed chromatin (69).

Alterations in the RB-E2F signaling pathway are known to be found in virtually all cases of OS, showing its importance in this tumor development. It is also known that lymphoid-specific helicase (HELLS) participates as a critical effector of chromatin remodeling downstream of the RB-E2F signaling pathway in various cancers, and has its expression regulated by the RB-E2F pathway. A study using an OS model in genetically modified mice revealed that the loss of the E2F1 and E2F3 transcription factors significantly delays tumor progression and increases the overall survival of mice with p53/Rb1 deficient OS. On the other hand, it has been seen that HELLS mRNA is upregulated and its protein is overexpressed in OS, but has no effect on tumor proliferation and migration. In addition, loss of HELLS in OS has no effect on tumor onset and overall survival of mice. The authors concluded that while HELLS may serve as a biomarker for tumorigenesis and for RB-E2F pathway status, it is unlikely to serve as a target for therapeutics in OS (93).

\section{Role of non-coding RNAs}

Current knowledge reveals that most of the genes that make up the human genome are transcribed into non-coding RNAs (ncRNAs) which play important roles in the normal functioning of the cell, but are also associated with pathological processes, including cancer and infectious diseases $(94,95)$. Although ncRNAs are not translated into protein, they perform important regulatory functions within the cell, and today are recognized as causing huge changes in all fields of biology and medicine due to its role in gene expression regulation (96). Increasing evidence has shown that ncRNAs, including miRNAs, non-coding long RNAs (lncRNAs) and circular RNAs (circRNAs) play important roles in regulating a wide range of biological processes involved in human disease etiology, including tumors (94). Some aspects related to these non-coding RNAs in OS development are subsequently presented.

MicroRNAs. MicroRNAs (miRNAs) are a class of small non-coding RNA endogenous containing 20-30 nucleotides 
which play important regulatory roles in various biological processes including differentiation, cell proliferation, cell cycle control, apoptosis, embryonic development and innate immunity $(97,98)$. miRNAs most often interact with the 3'untranslated regions (3'UTR) of target mRNAs to induce their mRNA degradation or translational repression. The interaction of miRNAs with other regions, including the 5'UTR gene promoter sequence, has also been reported. In addition, miRNAs may also activate translation or regulate transcription under certain conditions (99).

Some miRs are implicated in OS and may act as a factor protection or contribute to tumor initiation and progression. Evidence of this was obtained in an in vitro and in vivo functional validation study in tumor cell lines obtained in which the tumor suppressor role of miR-16 and the pro-metastatic role of miR-27a were confirmed (20).

It has been demonstrated that low levels of miR-200b expression have been associated with advanced clinical stage and metastasis in OS, and that its expression is down-regulated in tumor-derived U2OS, Saos2, HOS, and MG63 cell lines compared to normal osteoblasts. Restoring miR-200b expression led to a significant decrease in proliferation, migration, and invasion of tumor cells. In addition, ZEB1 gene encoding is a transcription factor which suppresses the interleukin 2 (IL-2) gene in specific T lymphocytes. It was identified as miR-200b target and its expression were down-regulated by miR-200b in OS. ZEB1 expression has also been shown to be significantly increased in tumor cells, while inhibition of ZEB1 expression has reduced proliferation, migration, and invasion of tumor cells. The results show that miR-200b inhibits proliferation of migration and invasion of tumor cells by inhibiting ZEB1 expression (100).

A recent study in MG-63 cells lines derived of OS showed that overexpression of miR-101 significantly suppressed the expression of ROCK1, a gene encoding a serine/threonine kinase signaling protein compared to knockdown of miR-101 in MG-63 cells. Overexpression of miR-101 reduced the viability, migration, and invasion of MG-63 cells and promoted apoptosis. Independent inhibition of $R O C K 1$ and reduction of miR-101 expression levels increased proliferation, migration, and invasion of MG-63 cells and inhibited apoptosis. In addition, the miR-101 inhibitory effect upon proliferation, migration, and invasion of MG-63 cells, and the activation of apoptosis were reversed in knockdown of ROCK1 in MG-63 cells. These results show that miR-101 plays a tumor suppressor role in OS by targeting the ROCK1 gene, and that overexpression of miR-101 inhibits tumor growth and tumor cell movement by inactivating the PI3K/AKT and JAK/STAT signaling pathways by down-regulation of $R O C K 1$ gene expression (Fig. 2) (101).

In another essay with cell lines derived from human OS and normal osteoblasts, transfection for up-regulation or down-regulation was used to measure the expression miR-3928. It was found that miR-3928 inhibited tumor growth, induced cell apoptosis, increased the percentage of cells in the G1 phase, and decreased the percentage of cells in the S phase in the up-regulation condition, whereas it promoted cellular proliferation and tumor growth in the down-regulation condition. This suggests that miR-3928 acts as a tumor suppressor, having the $E R B B 3, I L-6 R$, and $C D K 6$, gene encoders of the tyrosine-protein kinase receptor, IL-6 receptor, and cyclin-dependent kinase 6 as targets, respectively (Fig. 2) (102).

It has been previously reported that pulmonary metastasis formation in OS is inversely correlated with Fas (a type II transmembrane protein of the TNF family) expression on the cell surface. Interestingly, expression levels of miR-17-92 group members, including miR-20a and miR-19a, were observed to be higher in LM7 lineage metastatic cells expressing low Fas when compared to non-metastatic lines which present high Fas expression levels. An inverse correlation between Fas expression and miR-20a was observed in all analyzed tumor-derived cells. Overexpression of miR-20a resulted in a consistent and sustained negative regulation of Fas expression in SAOS-2 cells. Inhibition of miR-20a in LM7 cells increased Fas expression levels and reduced metastasis in mice injected with LM7 stably transfected with anti-miR-20a. This suggests that miR-20a encoded by the miR-17-92 gene negatively regulates Fas expression in OS, increasing its metastatic potential (Fig. 2) (103).

Another miR strongly associated with OS development is $\mathrm{miR}-574-3 p$, whose expression levels are increased very much in the tissue obtained from tumors, as well as in U2OS, SAOS, and MG63 OS-derived cell lines compared to normal osteoblasts. Negative regulation of miR-574-3p by antisense mi-574-3p resulted in cell growth inhibition and induced cellular apoptosis. Furthermore, overexpression of miR-574-3p by transfection with miR-574-3p mimics promoted a proliferation of U2OS cells. Functional analysis identified the decapentaplegic homologue 4 (SMAD4), which encodes a family of signaling proteins, is a target of miR-574-3p, since this gene function was suppressed in miR-574-3p transfected cells. It has also been shown that overexpression of SMAD4 was able to neutralize the promoter effects of miR-574-3p on the growth of cancer cells. Thus, it has been established that miR-574-3p exerts a tumor-promoting function in OS by down-regulating the expression of the SMAD4 tumor suppression gene (Fig. 2) (104).

In 40 OS tissue samples it was shown that $m i R-140$ expression is reduced and that restoration of its expression in OS-derived cells has a marked effect on inhibiting cell proliferation and invasion, inducing apoptosis in vitro, and suppressing tumor growth in vivo. A bioinformatics study revealed that miR-140 has the gene encoding histone deacetylase 4 (HDAC4) as target, and in this case $m i R-140$ acts as a tumor suppressor gene (105). Another study involving 85 patients with resectable OS and 56 patients with un-resectable OS showed a shorter disease-free survival in patients with low levels of expression of $m i R-125 b$. The low miR-125b expression was associated with advanced tumors in patients with un-resectable OS. The results suggest that low expression of circulating $m i R-125 \mathrm{~b}$ may be a potential marker of poor prognosis in patients with OS (106).

In OS metastatic cell models obtained by exogenous transfection of F5M 2 cells, a low level of $m i R-150$ expression and significantly increased Ezrin (a gene encoding the protein-tyrosine kinase) expression were found. The exogenous transfection of miR-150 mimics in F5M2 cells resulted in reduced Ezrin gene expression. In addition, overexpression of this gene has been shown to promote a significant suppression of the invasion and metastasis capability of F5M2 cells. 


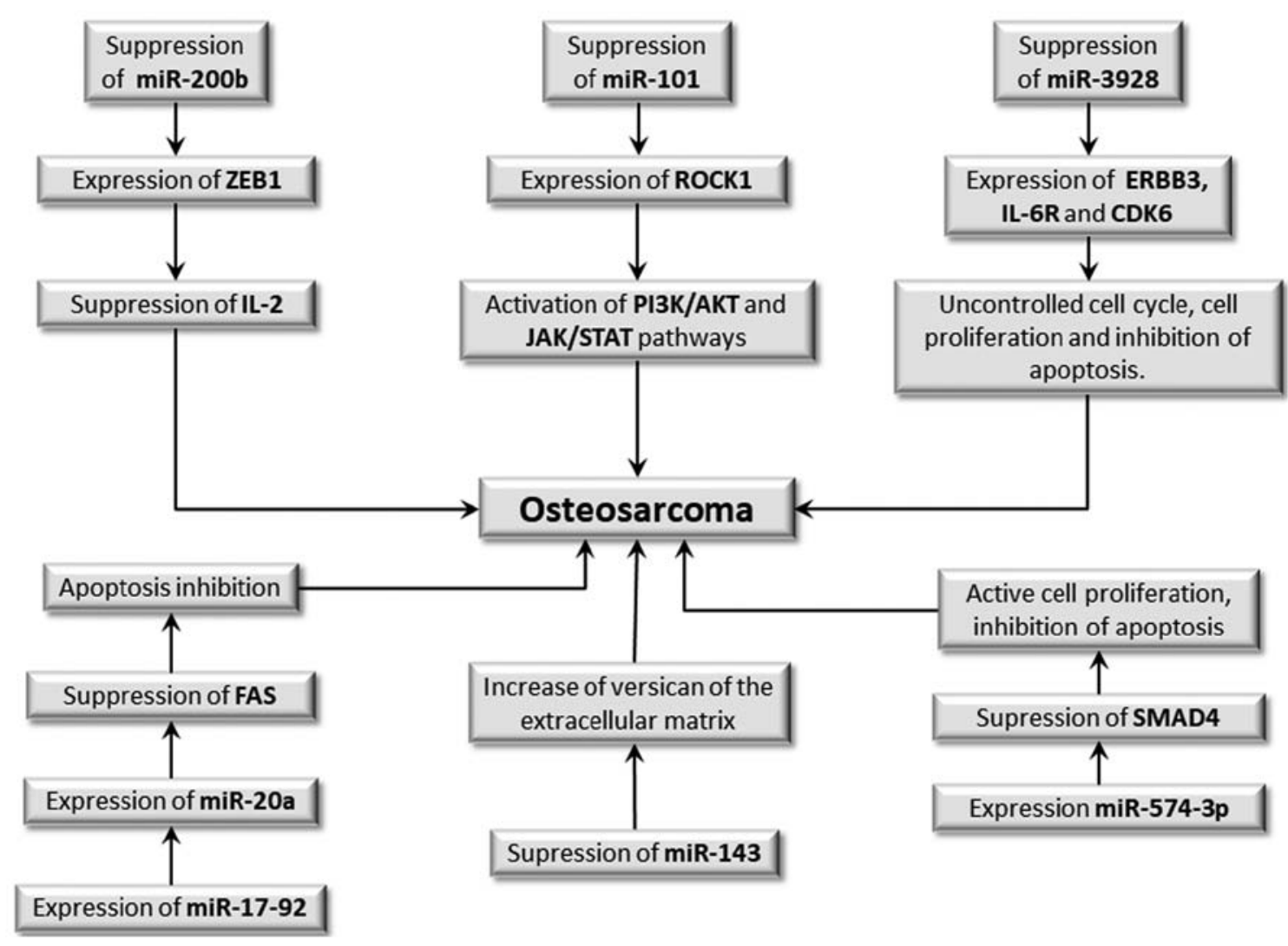

Figure 2. Participation of miRs in OS development. The suppression or activation of miR expression is an epigenetic event which may be involved in OS development. Suppression of miR-200b results in overexpression of the ZEB1 gene, whose product suppresses IL-2 gene expression, whereas the suppression of miR-101 results in overexpression of the ROCK1 gene which actuates in the PI3K/AKT and JAK/STAT signaling pathways. Both cases may increase the risk of initiation and progression of OS, since they cause impairment of the immune response. Suppression of miR-3928 increases the expression of the ERBB3, IL-6R and CDK6 genes, which may favor tumor development, promoting cell proliferation, uncontrolled cell cycle and apoptosis inhibition. The suppression of miR-143 results in the expression of versican and proteoglycan of extracellular matrix, favoring tumor progression. However, the expression of miR-17-92 increases the expression of miR-20a, which in turn reduces Fas expression, a cell death receptor, and inhibits apoptosis, whereas miR-574-3p expression suppresses expression of the SMAD4 tumor suppressor gene, which leads to increased cell proliferation and apoptosis inhibition. Therefore, both mechanisms may increase the risk of OS. miR, microRNA; OS, osteosarcoma.

The upregulation of $m i R-150$ results in down-regulation of Ezrin, which leads to a reduction in the invasion and metastasis capacity of tumor cells, indicating that miR-150 acts as a tumor suppressor in OS (107).

It was found that that miR-449c is significantly down-regulated in OS cells and presented hypermethylation of the DNA in two $\mathrm{CpG}$ islands adjacent to the miR-449c genomic locus in OS cells. Ectopic expression of miR-449c significantly inhibited OS cell proliferation, colony formation and caused cell cycle arrest in the G1 phase. miR-449c was able to negatively regulate c-Myc oncogene expression. On the other hand, overexpression c-Myc partially reversed cell proliferation and colony formation inhibited by miR-449c. This shows that miR-449c acts as a tumor suppressor, inhibiting c-Myc expression and that, in the OS miR-449c is down-regulated due to DNA methylation (83).

Long non-coding RNAs (lncRNAs). LncRNAs are transcribed with more than 200 nucleotides that play critical roles in different biological processes such as cell growth, transcription, and translation, epigenetic regulation of gene expression, splicing, nuclear cytoplasmic traffic, and cell cycle control (108). Recent studies show that IncRNAs can epigenetically regulate oncogenesis, which can prevent (109) favoring initiation and progression of OS $(110,111)$. Thus, IncRNAs can contribute to the development and progression of OS by acting as tumor suppressors or as oncogenes inducing tumor formation $(109,111)$. Therefore, they can modulate cancer pathogenesis in many aspects including proliferation migration, metastasis, invasion and cellular apoptosis (108).

It has been seen that lncRNAs can regulate OS by at least two mechanisms that target mRNA: By activating signaling pathways or by acting as a miRNA sponge. Positive regulation of the Hedgehog $(\mathrm{Hh})$ signaling pathway, which is implicated in the regulation of differentiation, proliferation, cell polarity and carcinogenesis, has been shown to promote the expression of $Y A P l$, a candidate human oncogene in multiple tumors, which in turn is responsible for aberrant expression of lncRNA H19 in malignant OS. In addition, IncRNAs may also regulate gene expression at post-transcriptional levels, acting as an endogenous 'sponge' and under regulation of a microRNA chain (108).

Several lncRNAs have been reported as important regulators in initiating and progressing OS, among them MALAT1 which has been found to be upregulated in tumor tissues compared to adjacent non-tumor soft tissues. Overexpression of this lncRNA results in tumor cell proliferation, migration and invasion in vitro and enhances tumor growth in a mouse 
xenograft model, and has also been correlated with poor prognosis. In OS, MALAT1 modulates RET proto-oncogene expression by sponging miR-129-5p, increasing protein expression downstream of the RET-Akt pathway, and its expression was positively correlated with $R E T$ and negatively correlated with miR-129-5p in original OS and in xenografted tumor cells. This shows that MALAT1 act as oncogenic lncRNA in OS by regulating RET via miR-129-5p suppression, thereby activating the PI3K-Akt signaling pathway (112).

One study shows that lncRNA HOXD-AS1 encoded by HOXD genes was significantly over-regulated in OS tissues and cells derived from this tumor, and that its overexpression was associated with poor prognosis of OS patients. HOXD-AS1 silencing resulted in inhibition of tumor cell proliferation, induced cycle arrest in the G1/G0 phase in vitro, and suppressed tumor cell growth in vivo. It was confirmed that HOXD-AS1 could interact with homologous zest enhancer (EZH2) of the p57 gene promoter, inhibiting its tumor suppressive action, favoring OS oncogenesis (113). It has also been shown that expression of the TUGl gene encoding lncRNA taurine 1 (TUG1) was significantly higher in tumor tissues than in adjacent normal bone tissues. Overexpression of TUG1 results in down-regulation of miR-212-3p expression causing increased tumor size, advanced lymph node metastasis, and decreased overall survival time of OS patients (114).

Another non-coding long RNA involved in initiating and progressing OS is lncRNA SNHG1, having miRNA-101-3p as targets since it is up-regulated, while miRNA $101-3 p$ is down-regulated in tumoral tissue and tumor-derived cell lines. In addition, IncRNA SNHG1 knockdown resulted in cell apoptosis and kept the cell cycle in the G0/G1 phase, with reduced overall cell viability. Under normal conditions, miRNA-101-3p acts by suppressing proliferation, migration and cell invasion. Thus, down-regulated expression of miRNA-101-3p enhances the expression of Rho-associated coiled-coil-containing protein kinase 1 (ROCK1) and promotes cell proliferation, migration and invasion. Overexpression of lncRNA SNHG1 results in inactivation of the phosphoinositide 3-kinase/ATK pathway and activation of the epithelial-mesenchymal transition of the OS-derived cell lines. Thus, lncRNA SNHG1 behaves as an oncogene, while miRNA-101-3p acts as a tumor suppressor (110).

On the other hand, RNA-steroid receptor RNA activator 1 (SRA1) plays a protective role against OS in targeting miRNA-208a, since its expression is down-regulated in tumor tissues compared to normal bone tissue, while expression of microRNA-208a was up-regulated in the tumor tissues. In addition, restoration of expression of this lncRNA inhibited proliferation, migration and invasion of tumor cells by increasing the apoptosis rate of these cells. Up-regulation of microRNA-208a played a similar role in silencing RNA-steroid receptor RNA activator 1, leading to inhibition of apoptosis and increasing tumor cell proliferation, migration and invasion (109).

Circular RNAs (circRNAs). Circular RNAs (circRNAs) are a class of endogenous non-coding RNAs generated from back-splicing, which are covalently closed in forming a circular loop structure, with high stability that can act in gene regulation $(115,116)$. Recent studies show that such molecules can regulate transcriptional or post-transcriptional gene expression by acting as miRNA sponges and are involved in the regulation of many important biological processes (117). CircRNAs have been shown to play a critical role in regulating gene expression in eukaryotes and therefore may play central roles in initiating and progressing cancer in humans (118).

In a microarray-based circRNA expression study performed on OS-derived cell lines and compared to normal cells, 12 differentially expressed circRNAs were found; among them, up-regulated hsa_circRNA_103801 and down-regulated hsa_circRNA_104980. The potential targets of hsa_ circRNA_103801 include hsa-miR-370-3p, hsa-miR-338-3p and hsa-miR-877-3p, while the potential targets of hsa circRNA_104980 were hsa-miR-1298-3p and hsa-miR-660-3p. Functional analysis showed that hsa_circRNA_103801 was involved in cancer signaling pathways such as HIF-1, VEGF and angiogenesis pathway, the Rap1 signaling pathway and the PI3K-Akt signaling pathway, while hsa_circRNA_104980 was related to some pathways such as the tight junction pathway (Fig. 3) (119).

The high expression of CircTADA2A was found in both OS tissue and tumor-derived cell lines. The inhibition of circTADA2A expression attenuated tumor cell proliferation, migration and invasion in vitro, as well as tumorigenesis and metastasis in vivo. It has been shown that circTADA2A acts as a sponge, absorbing miR-203a-3p to regulate $C R E B 3$ expression, which has been identified as an OS-conducting gene. In addition, the inhibition miR-203a-3p, or CREB3 overexpression could reverse the circTADA2A silencing-induced impairment in malignant tumor behavior (Fig. 3) (118).

\section{Role of cytokines}

Cytokine is a generic term used to denote a large group of signaling proteins secreted by specific cells in response to stressful conditions which mediate and regulate immunity, inflammation, and hematopoiesis. Such molecules are also designated as the basis in their presumed function, secretion cell, or target of their action. For example, cytokines produced by lymphocytes may be referred to as lymphokines, which are also known as interleukins (ILs), since they are not only secreted by leukocytes, but are also capable of affecting leukocyte cellular responses (120).) Although the main function of cytokines is to seek homeostasis in conditions of stress and tissue damage when there are failures in this process and the stressful condition remains for a long time, the persistence of cytokines will increase the risk of malignant transformation. Thus, host responses to stress can affect various stages of cancer initiation and tumor progression (121). Therefore, it is very important to understand the deep and complex interaction between the different cytokines in the oncogenesis process, including those which occur in OS development (23). Next, we present some cytokines whose actions are cited as possible mechanisms involved in OS development.

Interleukin $6(I L-6)$. IL-6 is among the possible cytokines involved in OS development, and is a pro-inflammatory cytokine which activates Janus kinase (JAK), promoting the phosphorylation of transcription activator 3 (STAT3), which 


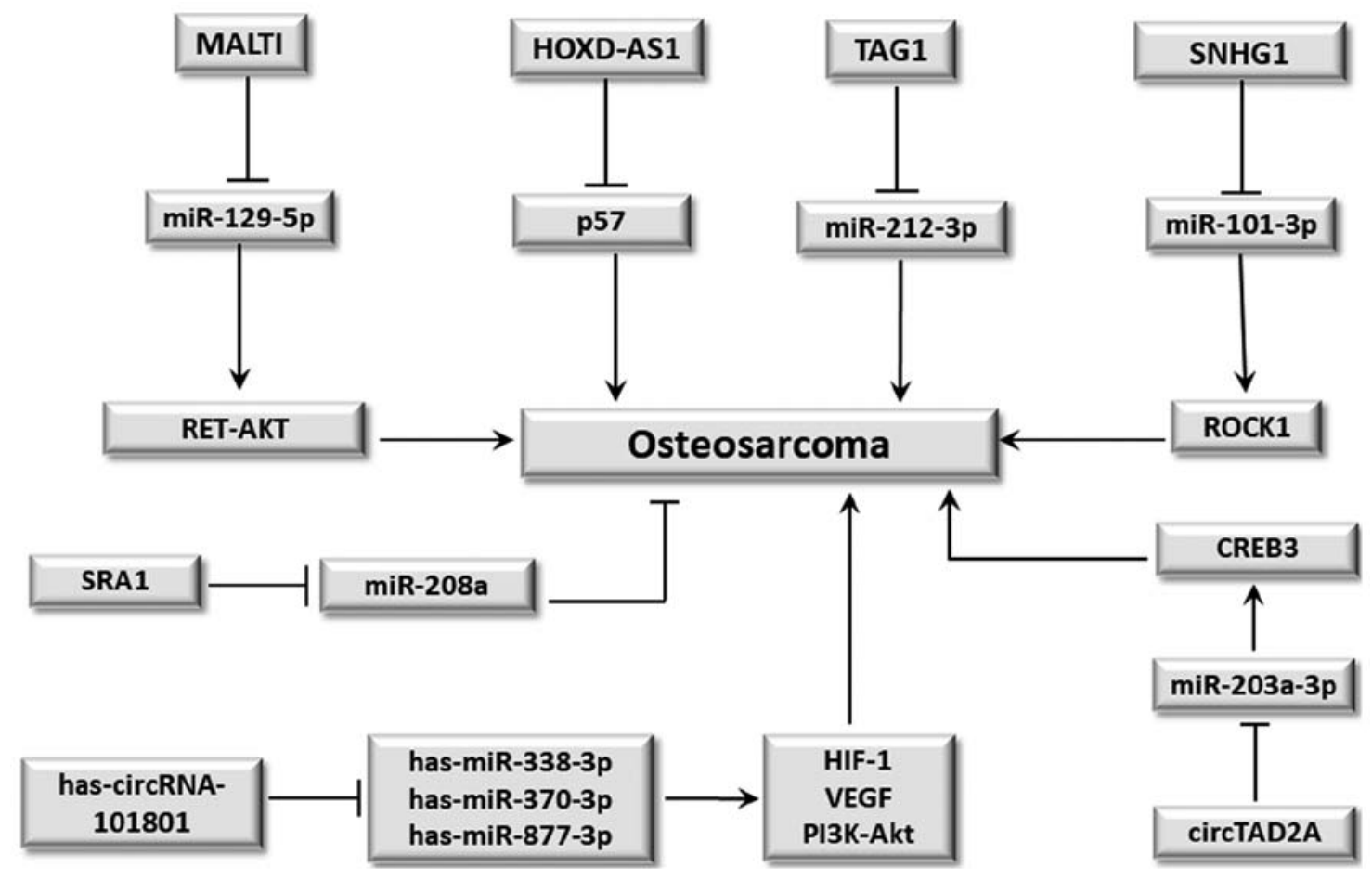

Figure 3. Role of lncRNAs and circRNAs in osteosarcoma. In general, lncRNAs and circRNAs act as sponges for miRNAs, causing its inactivation and favoring tumor development. Therefore, MAULTI acts by suppressing miR-129-5p which leads to activation of the RET-AKT signaling pathway; HOXD-AS1 suppresses the tumor suppressing action of 57 protein; TAG1 suppresses miR-212-3p, whereas SNHG1 suppresses miR-101-3p, which results in activation of ROCK1 gene expression. All these events favor the development of osteosarcoma. However, lncRNA SRA1 acts by suppressing miR-208a, inhibiting its tumorigenic action. Additionally, has-circRNA-101801 circRNA acts by suppressing miRNAs: has-miR-338-3p, has-miR-370-3p and has-miR-877-3p resulting in increased expression of HIF-1 and VEGF, and PI3K-Akt signaling pathway activation, thus favoring angiogenesis. Furthermore, circRNA TAD2A suppresses miR-203a-3p, which leads to increased CREB expression. This favors tumor development in both cases. circRNA, circular RNA; lncRNA, long non-coding RNA.

in turn signals for increased cell proliferation and inhibits apoptosis of the MSCs and of OS-derived cells (122). High expression levels of SOX18, IL-6 and p-STAT3 are found in OS, compared with normal bone tissue (123). It has been shown that neutralization of IL- 6 with antibody or by the STAT3 inactivation reduces tumor progression, besides inhibiting JAK2 preventing lung metastases and increasing survival in animals (122). In addition, IL-6 contributes to bone degradation by promoting the osteoclast differentiation and expression of proteins which act on bone resorption and induces the expression of the vascular endothelial growth factor (VEGF) in OS cells $(124,125)$. Additionally, IL-6 can function as a mediator of pulmonary tropism of cell of the OS favoring the installation of metastases in these organs (126).

Transforming growth factor beta (TGF $\beta)$. TGF $\beta$ is linked to the dedifferentiation of MSCs in OS, a dynamic population of cells associated with tumor invasion and radio-and chemoresistance with poor prognosis (127). TGF $\beta$ is produced by autocrine signaling of cancer cells which enhance the migration potential of OS cells through the activation of the MAPK pathway (128). Activation of TGF $\beta$ signal transduction activates pleiotropic functions involved in regulating cell proliferation and differentiation, apoptosis, cell migration and invasion, extracellular matrix production, angiogenesis and immune response $(129,130)$.

Due to its complex activity, TGF $\beta$ plays an ambiguous role in tumors in humans. It acts as a tumor suppressor in the early stages of tumorigenesis, inhibiting cell proliferation and immortalization, and promoting apoptosis. In later stages it promotes metastasis, migration, invasion and chemotaxis, and its functions are associated with aggressive and invasive tumors $(131,132)$. Regarding OS, it was demonstrated in vitro that tumor cells secrete TGF $\beta$ by activating the TGF $\beta /$ SMAD-2/-3 signaling pathway, keeping MSCs in an undifferentiated state and producing higher levels of pro-tumor cytokines such as IL- 6 and VEGF (28). High TGF $\beta$ mRNA levels were also reported in OS-derived cells and are associated with aggressive behavior and lung metastases (133). In addition, an association was observed between a significant increase in the activation of SMAD3 signaling pathway and high TGF $\beta 1$ levels in serum with a higher risk of developing lung metastasis in OS patients (28).

An in vitro assay showed that TGF- $\beta 1$ promoted OS cell migration and invasion by up-regulating the expression of versican, an extracellular matrix proteoglycan, whose expression is down-regulated by miR-143. TGF- $\beta$ mechanistically activates the MAPK pathway, which in turn activates the TGF- $\beta /$ SMAD-2/-3 pathway, leading to miR-143 suppression which leads to an increase of versican in the extracellular matrix, thus contributing to tumor progression since this favors tumor cell migration and invasion (Fig. 4) (129).

TGF- $\beta$ signaling under hypoxia conditions dramatically increases the self-renewal capacity of MSCs in OS, resulting in an increased potential for tumorigenesis, neovasculogenesis, and metastasis. The blockade of TGF- $\beta 1$ signaling inhibited 


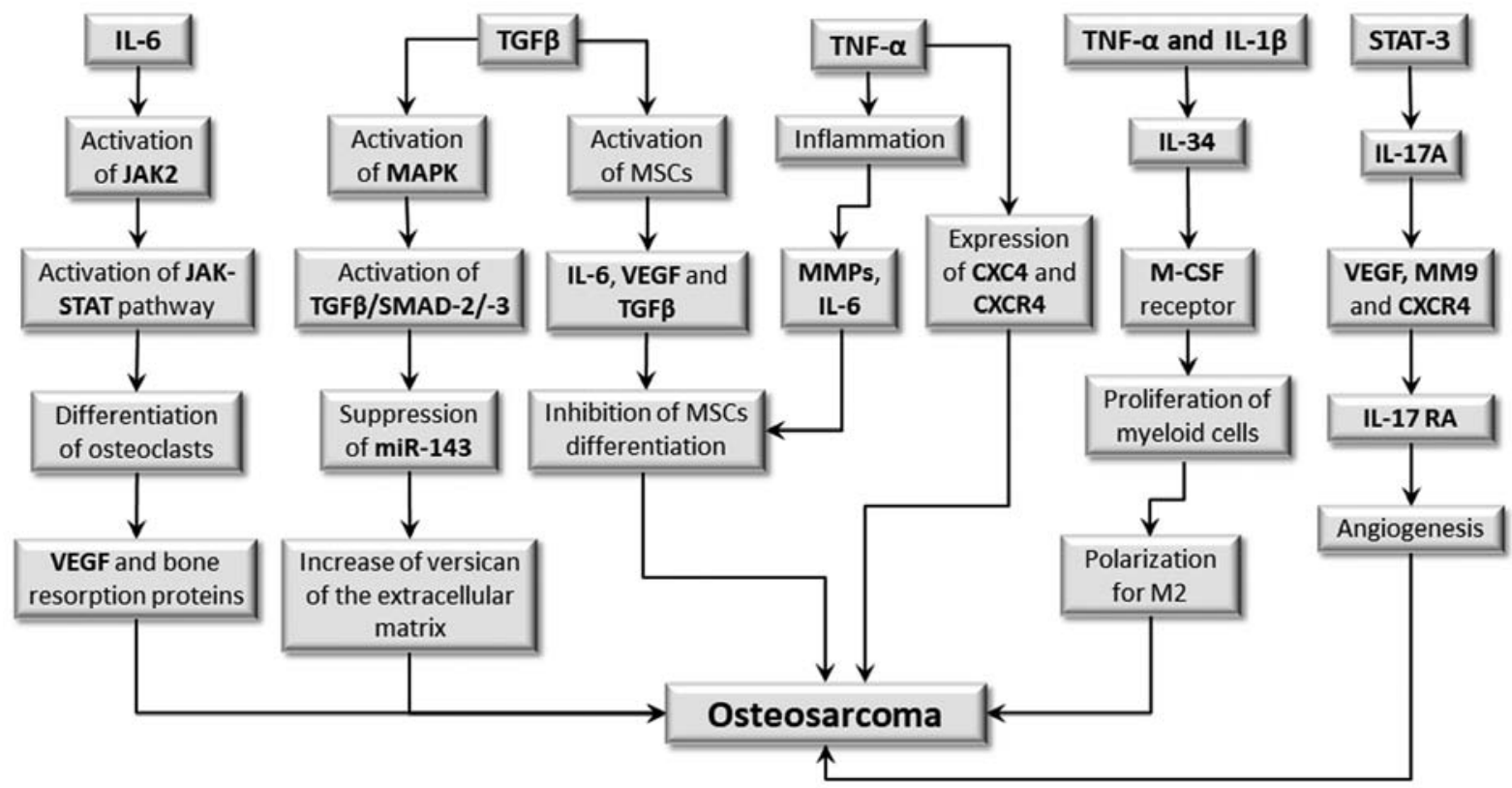

Figure 4. Role of cytokines in human OS. Certain cytokines may contribute to OS development by activating cell signaling pathways, or by interfering in the differentiation process of MSCs. IL-6 activates the expression of JAK2 signal transducer, which in turn activates JAK-STAT signaling pathway that induces the differentiation of MSCs into osteoclasts. This leads to the production of VEGF and of proteins which act on bone resorption and induces angiogenesis, contributing to tumor development. TGF $\beta$ activates the MAPK pathway, which in turn activates the TGF- $\beta /$ SMAD- $2 /-3$ pathway, resulting in miR-143 suppression, with a consequent increase in versican expression of the extracellular matrix, thus contributing to tumor progression. TGF- $\beta$ also acts on MSCs, inducing the production of IL-6, VEGF and additional TGF- $\beta$. IL-6 together with TNF $\alpha$ induces inflammation and the production of MMPs which (in synergistic action) inhibits MSC differentiation, increasing the risk of initiating OS. Furthermore, TNF $\alpha$ also increases the expression of CXC4 chemokine and its CXCR4 receptor, a condition which also favors tumor development. TNF $\alpha$ together with IL1 $\beta$ activates the production of IL-34 which binds to the M-CSF receptor, promoting growth and survival of myeloid cells and macrophage polarization for the M2 profile with recruitment of these cells into the tumor environment, therefore contributing to its progression. The STST3 transcription factor, upon being phosphorylated, activates cytokine IL-17 production, which (if connected to its IL-17RA receptor) stimulates VEGF, MM9 and CXCR4 production and promotes angiogenesis, thus contributing to tumor progression and formation of metastases. MSCs, mesenchymal stem cells; OS, osteosarcoma.

the differentiation and clonogenicity of tumor cells and reduced hypoxia-mediated self-renewal of MSCs. These findings suggest that a dynamic balance exists between stem cells and non-stem cells within the cell population of the OS, and that MSCs can be generated from differentiated cancer cells (127).

It has been shown that the Saos-2 and U2-OS cell lines derived from OS produce high TGF $\beta$ levels as they activate MSCs to produce IL-6 and VEGF, inhibiting the osteogenic differentiation of MSCs. In addition, treatment with the anti-TGF- $\beta$ antibody significantly reduced the IL- 6 and VEGF production by MSCs and induced their osteogenic differentiation, showing that TGF $\beta$ plays an important role in tumor initiation (28). In an orthotopic xenograft mouse model of OS, tumor cells have been shown to incorporate TGF $\beta$ in the form associated with membrane, which induces IL-6 production by tumor mesenchymal stem cells promoting tumor growth, accompanied by the intratumor activation of STAT3 and formation of pulmonary metastases (Fig. 4) (132). In addition, TGF $\beta$ induces mesenchymal epithelial transition by inhibiting of miR-499a expression, interacting with the Snaill/Zeb1 of miR-499a promoter. This result in phenotypic conversion of primary tumor cells that acquire the ability to migrate and generate pulmonary metastases (134).

Tumor necrosis factor (TNF- $\alpha$ ). TNF- $\alpha$ is a pro-inflammatory cytokine produced by lymphocytes and macrophages which, although it can induce apoptosis of tumor cells, is associated with progression of several types of tumors, including OS (29).
TNF- $\alpha$ increases pulmonary metastasis in OS by increasing CXC 4 (CXCR4) chemokine receptor expression. In a mouse model, infliximab treatment, a TNF- $\alpha$ inhibitor decreased CXCR4 expression and significantly reduced cellular mobility and lung metastases (135). In an OS murine model induced by the transfer of AX MSCs of INK4a-deficient to wild type mice, the production of NF- $\alpha$ resulted in tumor growth and maintaining cells in the undifferentiated state by means of extracellular signal-regulated protein kinases (136). The treatment with TNF- $\alpha$ inhibitor resulted in reduced tumor growth, increased osteoblast differentiation, and the survival of the animals, highlighting the pro-tumorigenic effect of TNF- $\alpha$ on OS (29).

Interleukin 34 (IL-34). IL-34 has recently been identified and characterized by its ability to form macrophage colonies in human bone marrow cell cultures, constituting a similar function to that of the macrophage colony stimulating factor (M-CSF) including its synergistic action on inflammation (137). IL-34 signaling occurs by its binding to the M-CSF receptor, which is expressed in human mononuclear phagocytes (138). Similarly, to M-CSF, IL-34 stimulates growth and survival of myeloid cells and induces macrophage polarization to the profile of M2 tumor-associated macrophages $(139,140)$.

High levels of IL-34 expression are reported in several types of cancers and are associated with poor prognosis. In OS, IL-34 increases the blood supply, recruits and polarizes macrophages to the M2 profile functioning as an angiogenic, 
pro-metastatic factor and stimulator of tumor progression. The paratibial inoculation of human OS cells which resulted in overexpressing IL-34 in a murine model revealed that this cytokine is correlated with tumor progression, promoting angiogenesis, tumor growth, and pulmonary metastasis formation. In addition, IL-34 promoted M2 recruitment to inside the tumor. It has also been shown that IL-34 is expressed in OS cells regulated by TNF- $\alpha$, IL-1 $\beta$, and contributes to tumor growth by promoting M2 macrophage recruitment (Fig. 4) (140).

Interleukin 17 (IL-17). IL-17 is a cytokine produced by Th17 cells and other cell types, including neutrophils, NK, TCD8+, and $\mathrm{T} \gamma \delta$ cells, whose role in cancer development is still controversial, although it is correlated with poor prognosis in many types of human cancers. In stromal cells, it has been shown that IL-17 induces angiogenesis stimulators including VEGF, and that IL-17 receptor expression is associated with VEGF production by OS-derived cell lines $(141,142)$. In the murine model, IL-17A interaction with its IL-17RA receptor has been shown to promote metastasis in nude mice (non-T-cell athymic) inoculated with OS-derived cell lines expressing high levels of IL-17RA and then transfected with IL-17 gene encoding (142). In addition, clinical trials have shown that patients with OS had higher IL-17 serum levels when compared to healthy subjects, and that IL-17 levels were even higher in patients with metastases (141).

IL-17RA expression was also higher in the tumor tissue of patients with metastatic OS and in the U-2 cell line derived from the tumor, but not in MG63 cells. Interestingly, negative IL-17RA regulation in U-2 cells was able to nullify the increase in IL-17A-induced metastasis, while upregulation of IL-17RA in MG63 cells increased the ability of these cells to produce metastasis in response to IL-17A. The increased metastasis may be due to the interaction of IL-17A with its IL-17RA receptor, resulting in increased VEGF, MMP9, and CXCR4 expression in tumor cells. In addition, STAT3 activity was shown to be crucial in the interaction of IL-17A/IL-17RA to promote metastasis in OS (Fig. 4) (142).

\section{Conclusions}

OS, is a disease of multifactorial origin which involves a complex interaction between a wide variety of factors and mechanisms that when acting together promotes the deregulation of cellular signaling pathways, causing disturbances in bone tissue homeostasis. Bone tissue renewal requires an intensification of the differentiation process of precursor cells into the bone formation. As most cases of OS begin on the bone growth plate, disturbances in differentiation of precursor cells play a role in tumor initiation. It is believed that MSCs, osteogenic precursor cells which give rise to osteoblasts for bone formation, are a key component in OS initiation. This hypothesis is reinforced by experimental evidence involving the premalignant stages of the disease through the functional and phenotypic parallel analysis of normal MSCs, transformed MSCs, and MSCs derived from the tumor. The karyotyping of different MSCs revealed the occurrence of aneuploidization, translocations, and homozygous loss of the Cdkn2 region of the genome of those cells which controls the CDKN2A/p16 singling pathway, and plays a crucial role in malignant transformation of MSCs.
Another important aspect is the changes in the functions of tumor suppressor genes and/or oncogenes, either due to the occurrence of mutations, interference of epigenetic mechanisms or even by the action of cytokines. Such events can alter the crosstalk between cells and promote changes in their behavior, leading to activation or deactivation of signaling pathways which regulate cellular processes such as differentiation, proliferation, migration, and apoptosis, increasing the risk of suffering malignant transformation. Experimental evidence obtained through an in vivo study in transgenic animals in which the TP53 and RBI genes of MSCs cells were silenced reinforced the importance of the dysfunction of these genes in the development of OS. In addition, expression deregulation of tumor suppressor genes and of other genes, including oncogenes, may also occur due to epigenetic mechanisms triggered by environmental factors. These events influence gene activation and silencing, and are frequently found in cells obtained from tumors. Epigenetic mechanisms such as DNA methylation, histone modifications, nucleosome remodeling, and the action non-coding RNAs are often implicated in OS progression. It is believed that epigenetic mechanisms act individually or together to change the expression of tumor suppressor genes and/or oncogenes, activating or deactivating the transcription of these genes, resulting in the deregulation of cell signaling pathways which, in some way, triggers the tumor initiation and progression process.

Major scientific and conceptual advances have been made in recent years, especially in the field of biology, focusing on the mechanisms of tumor initiation, progression, metastasis and heterogeneity. This is due to technological advances which have enabled developing experimental models of tumors that mimic the disease in humans. Along with the growing knowledge about the mechanisms involved in tumor pathogenesis, several researchers are devoting themselves to try to find ways to infer these mechanisms in order to discover new options for treating the disease. Thus, we believe that the prospects are very promising to achieve more advances soon, as well as in the clinical area.

\section{Acknowledgements}

Not applicable.

\section{Funding}

No funding was received.

\section{Availability of data and materials}

Not applicable.

\section{Authors' contributions}

JWVDA was involved in drafting the manuscript and was a major contributor in writing the manuscript. TAADMF was involved in literature review and revising the manuscript critically for important intellectual content, and was a major contributor in writing the manuscript. JVFJr was involved in drafting the manuscript and literature review. JCVDA was involved in literature review and revising the manuscript critically for important 
intellectual content. DCFL was involved in literature review and revising the manuscript critically for important intellectual content. CMB was involved in drafting the manuscript and literature review. VSA was involved in the conception of the study and revising the manuscript critically for important intellectual content. JMGDA was involved in literature review and drafting the manuscript. JVF was involved in the conception and design of the study, drafting the manuscript and was the major contributor in writing the manuscript. All authors have read and approved the manuscript.

\section{Ethics approval and consent to participate}

Not applicable.

\section{Patient consent for publication}

Not applicable.

\section{Competing interests}

The authors declare that they have no competing interests.

\section{References}

1. Klein MJ and Siegal GP: Osteosarcoma: Anatomic and histologic variants. Am J Clin Pathol 125: 555-581, 2006.

2. Stiller CA, Desandes E, Danon SE, Izarzugaza I, Ratiu A, Vassileva-Valerianova Z and Steliarova-Foucher E: Cancer incidence and survival in European adolescents (1978-1997)-report from the automated childhood cancer information system project. Eur J Cancer 42: 2006-2018, 2006.

3. Nie $\mathrm{Z}$ and Peng H: Osteosarcoma in patients below 25 years of age-An observational study of incidence, metastasis, treatment and outcomes. Oncol Lett 16: 6502-6514, 2018.

4. Ottaviani G and Jaffe N: The epidemiology of osteosarcoma. Cancer Treat Res 152: 3-13, 2009.

5. Geller DS and Gorlick R: Osteosarcoma: A review of diagnosis, management, and treatment strategies. Clin Adv Hematol Oncol 8 : 705-718, 2010.

6. Morrow JJ and Khanna C: Osteosarcoma genetics and epigenetics: Emerging biology and candidate therapies. Crit Rev Oncog 20: 173-197, 2015.

7. Rao-Bindal K and Kleinerman ES: Epigenetic regulation of apoptosis and cell cycle in osteosarcoma. Sarcoma 2011: 679457, 2011.

8. Mutsaers AJ and Walkley CR: Cells of origin in osteosarcoma: Mesenchymal stem cells or osteoblast committed cells? Bone 62 : $56-63,2014$.

9. Zheng Y, Wang G, Chen R, Hua Y and Cai Z: Mesenchymal stem cells in the osteosarcoma microenvironment-their biological properties, influence on tumor growth, and therapeutic implications. Stem Cell Res Ther 9: 22, 2018.

10. Alfranca A, Martinez-Cruzado L, Tornin J, Abarrategi A, Amaral T, de Alava E, Menendez P, Garcia-Castro J and Rodriguez R: Bone microenvironment signals in osteosarcoma development. Cell Mol Life Sci 72: 3097-3113, 2015.

11. Velletri T, Xie N, Wang Y, Huang Y, Yang Q, Chen X, Chen Q, Shou P, Gan Y, Cao G, et al: P53 functional abnormality in mesenchymal stem cells promotes osteosarcoma development. Cell Death Dis 7: e2015, 2016.

12. Han Y, Kim YM, Kim HS and Lee KY: Melatonin promotes osteoblast differentiation by regulating Osterix protein stability and expression. Sci Rep 7: 5716, 2017.

13. Komori T: Runx2, an inducer of osteoblast and chondrocyte differentiation. Histochem Cell Biol 149: 313-323, 2018.

14. Shin MH, He Y, Marrogi E, Piperdi S, Ren L, Khanna C, Gorlick R, Liu C and Huang J: A RUNX2-mediated epigenetic regulation of the survival of p53 defective cancer cells. PLoS Genet 12: e1005884, 2016.

15. Martin JW, Zielenska M, Stein GS, van Wijnen AJ and Squire JA: The role of RUNX2 in osteosarcoma oncogenesis. Sarcoma 2011: 282745, 2011.
16. Molchadsky A, Shats I, Goldfinger N, Pevsner-Fischer M, Olson M, Rinon A, Tzahor E, Lozano Gina G, Zipori D, Sarlig R and Rotter V: p53 plays a role in mesenchymal differentiation programs, in a cell fate dependent manner. PLoS One 3: e3707, 2008.

17. Tang N, Song WX, Luo J, Haydon RC and He TC: Osteosarcoma development and stem cell differentiation. Clin Orthop Relat Res 466: 2114-2130, 2008.

18. Martin JW, Squire JA and Zielenska M: The genetics of osteosarcoma. Sarcoma 2012: 627254, 2012.

19. Sharma S, Kelly TK and Jones PA: Epigenetics in cancer. Carcinogenesis 31: 27-36, 2010.

20. Jones KB, Salah Z, Del Mare S, Galasso M, Gaudio E, Nuovo GJ, Lovat F, LeBlanc K, Palatini J, Randall RL, et al: MicroRNA signatures associate with pathogenesis and progression of osteosarcoma. Cancer Res 72: 1865-1877, 2012.

21. Li Y, Meng G, Huang L and Guo QN: Hypomethylation of the P3 promoter is associated with up-regulation of IGF2 expression in human osteosarcoma. Hum Pathol 40: 1441-1447, 2009.

22. Lu J and Wang J: IRX1 hypomethylation in osteosarcoma metastasis. Oncotarget 6: 16802-16803, 2015.

23. Lopes-Júnior LC, Silveira DSC, Vulczak A, Santos JC, Veronez LC, Fisch A, Flória-Santos M, Lima RAG and Pereira-da-Silva G: Emerging cytokine networks in osteosarcoma. Oncol Commun 2: e1167, 2016.

24. Yang Y, Yang R, Roth M, Piperdi S, Zhang W, Dorfman H, Rao P, Park A, Tripathi S, Freeman C, et al: Genetically transforming human osteoblasts to sarcoma: Development of an osteosarcoma model. Genes Cancer 8: 484-494, 2017.

25. Broadhead ML, Clark JCM, Myers DE, Dass CR and Choong PFM: The molecular pathogenesis of osteosarcoma: A review. Sarcoma 2011: 959248, 2011.

26. Denduluri SK, Wang Z, Yan Z, Wang J, Wei Q, Mohammed MK, Haydon RC, Luu HH and He TC: Molecular pathogenesis and therapeutic strategies of human osteosarcoma. J Biomed Res 30: 5-18, 2016.

27. Deng ZL, Sharff KA, Tang N, Song WX, Luo J, Luo X, Chen J, Bennett E, Reid R, Manning D, et al: Regulation of osteogenic differentiation during skeletal development. Front Biosci 13: 2001-2021, 2008

28. Tu B, Peng ZX, Fan QM, Du L, Yan W and Tang TT: Osteosarcoma cells promote the production of pro-tumor cytokines in mesenchymal stem cells by inhibiting their osteogenic differentiation through the TGF- $\beta /$ Smad $2 / 3$ pathway. Exp Cell Res 320: 164-173, 2014.

29. Mori T, Sato Y, Miyamoto K, Kobayashi T, Shimizu T, Kanagawa H, Katsuyama E, Fujie A, Hao W, Tando T, et al: $\mathrm{TNF} \alpha$ promotes osteosarcoma progression by maintaining tumor cells in an undifferentiated state. Oncogene 33: 4236-4246, 2014.

30. Hanahan D and Weinberg RA: Hallmarks of cancer: The next generation. Cell 144: 646-674, 2011.

31. Tung PY and Knoepfler PS: Epigenetic mechanisms of tumorigenicity manifesting in stem cells. Oncogene 34: 2288-2296, 2015.

32. Klimczak A and Kozlowska U: Mesenchymal stromal cells and tissue-specific progenitor cells: their role in tissue homeostasis. Stem Cells Int 2016: 4285215, 2016.

33. Mohseny AB, Szuhai K, Romeo S, Buddingh EP, Briaire-de Bruijn I, de Jong D, van Pel M, Cleton-Jansen AM and Hogendoorn PC: Osteosarcoma originates from mesenchymal stem cells in consequence of aneuploidization and genomic loss of Cdkn2. J Pathol 219: 294-305, 2009.

34. Zhang D and Liu S: SOX5 promotes epithelial-mesenchymal transition in osteosarcoma via regulation of Snail. J BUON 22: 258-264, 2017.

35. Mannerström B, Kornilov R, Abu-Shahba AG, Chowdhury IM, Sinha S, Seppänen-Kaijansinkko R and Kaur S: Epigenetic alterations in mesenchymal stem cells by osteosarcoma-derived extracellular vesicles. Epigenetics 14: 352-364, 2019.

36. Chen Z, Guo J, Zhang K and Guo Y: TP53 mutations and survival in osteosarcoma patients: A meta-analysis of published data. Dis Markers 2016: 4639575, 2016.

37. Perry JA, Kiezun A, Tonzi P, Van Allen EM, Carter SL, Baca SC, Cowley GS, Bhatt AS, Rheinbay E, Pedamallu CS, et al: Complementary genomic approaches highlight the PI3K/mTOR pathway as a common vulnerability in osteosarcoma. Proc Natl Acad Sci USA 111: E5564-E5573, 2014.

38. Kovac M, Blattmann C, Ribi S, Smida J, Mueller NS, Engert F, Castro-Giner F, Weischenfeldt J, Kovacova M, Krieg A, et al: Exome sequencing of osteosarcoma reveals mutation signatures reminiscent of BRCA deficiency. Nat Commun 6: 8940, 2015. 
39. Wang D, Niu X, Wang Z, Song CL, Huang Z, Chen KN, Duan J, Bai H, Xu J, Zhao J, et al: Multiregion sequencing reveals the genetic heterogeneity and evolutionary history of osteosarcoma and matched pulmonary metastases. Cancer Res 79: 7-20, 2019.

40. Lin PP, Pandey MK, Jin F, Raymond AK, Akiyama H and Lozano G: Targeted mutation of p53 and Rb in mesenchymal cells of the limb bud produces sarcomas in mice. Carcinogenesis 30 : 1789-1795, 2009.

41. Rutkovskiy A, Stensløkken Ko and Vaage IJ: Osteoblast differentiation at a glance. Med Sci Monit Basic Res 22: 95-106, 2016

42. Long F: Building strong bones: Molecular regulation of the osteoblast lineage. Nat Rev Mol Cell Biol 13: 27-38, 2011.

43. Techavichit P, Gao Y, Kurenbekova L, Shuck R, Donehower LA and Yustein JT: Secreted Frizzled-Related Protein 2 (sFRP2) promotes osteosarcoma invasion and metastatic potential. BMC Cancer 16: 869, 2016.

44. Kim H, Yoo S, Zhou R, Xu A, Bernitz JM, Yuan Y, Gomes AM, Daniel MG, Su J, Demicco EG, et al: Oncogenic role of SFRP2 in p53-mutant osteosarcoma development via autocrine and paracrine mechanism. Proc Natl Acad Sci USA 115: E11128-E11137, 2018.

45. Chen KS, Kwon WS, Kim J, Heo SJ, Kim HS, Kim HK Kim SH, Lee WS, Chung HC, Rha SY and Hwang TH: A novel TP53-KPNA3 translocation defines a de novo treatment-resistant clone in osteosarcoma. Cold Spring Harb Mol Case Stud 2: a000992, 2016.

46. Del Mare S, Kurek KC, Stein GS, Lian JB and Aqeilan RI: Role of the WWOX tumor suppressor gene in bone homeostasis and the pathogenesis of osteosarcoma. Am J Cancer Res 1: 585-594, 2011.

47. Del Mare S and Aqeilan RI: Tumor Suppressor WWOX inhibits osteosarcoma metastasis by modulating RUNX2 function. Sci Rep 5: 12959, 2015.

48. Henley SA and Dick FA: The retinoblastoma family of proteins and their regulatory functions in the mammalian cell division cycle. Cell Div 7: 10, 2012

49. Abreu Velez AM and Howard MS: Tumor-suppressor genes, cell cycle regulatory checkpoints, and the skin. N Am J Med Sci 7: 176-188, 2015.

50. Min EY, Kim IH, Lee J, Kim EY, Choi YH and Nam TJ: The effects of fucodian on senescence are controlled by the p16INK4a-pRb and p14Arf-p53 pathways in hepatocellular carcinoma and hepatic cell lines. Int J Oncol 45: 47-56, 2014.

51. Shi D and Gu W: Dual roles of MDM2 in the regulation of $\mathrm{p} 53$ : Ubiquitination dependent and ubiquitination independent mechanisms of MDM2 repression of p53 activity. Genes Cancer 3: 240-248, 2012.

52. Zhang H, Mao JS and Hu WF: Functional genetic single-nucleotide polymorphisms (SNPs) in cyclin-dependent kinase inhibitor 2A/B (CDKN2A/B) locus are associated with risk and prognosis of osteosarcoma in chinese populations. Med Sci Monit 25: $1307-1313,2019$.

53. Zhao $\mathrm{Y}, \mathrm{Yu} \mathrm{H}$ and $\mathrm{Hu} \mathrm{W}$ : The regulation of MDM2 oncogene and its impact on human cancers. Acta Biochim Biophys Sin (Shanghai) 46: 180-189, 2014.

54. Yu Q, Li Y, Mu K, Li Z, Meng Q, Wu X, Wang Y and Li L: Amplification of Mdmx and overexpression of MDM2 contribute to mammary carcinogenesis by substituting for p53 mutations. Diagn Pathol 9: 71, 2014

55. Han G, Wang Y and Bi W: C-Myc overexpression promotes osteosarcoma cell invasion via activation of MEK-ERK pathway. Oncol Res 20: 149-156, 2012

56. Wu X, Cai ZD, Lou LM and Zhu YB: Expressions of p53, c-Myc, Bcl-2 and apoptotic index in human osteosarcoma and their correlations with prognosis of patients. Cancer Epidemiol 36: 212-216, 2012

57. Chen D, Zhao Z, Huang Z, Chen DC, Zhu XX, Wang YZ, Yan YW, Tang S, Madhavan S, Ni W, et al: Super enhancer inhibitors suppress MYC driven transcriptional amplification and tumor progression in osteosarcoma. Bone Res 6: 11, 2018.

58. Wang Q, Liu H, Wang Q, Zhou F, Liu Y, Zhang Y, Ding H, Yuan M, Li F and Chen Y: Involvement of c-Fos in cell proliferation, migration, and invasion in osteosarcoma cells accompanied by altered expression of Wnt 2 and Fzd9. PLoS One 12: e0180558, 2017.

59. Xu G, Guo Y, Xu D, Wang Y, Shen Y, Wang F, Lv Y, Song F, Jiang D, Zhang Y, et al: TRIM14 regulates cell proliferation and invasion in osteosarcoma via promotion of the AKT signaling pathway. Sci Rep 7: 42411, 2017.

60. Nowacka-Zawisza M and Wiśnik E: DNA methylation and histone modifications as epigenetic regulation in prostate cancer. Oncol Rep 38: 2587-2596, 2017.
61. Shanmugam MK, Arfuso F, Arumugam S, Chinnathambi A, Jinsong B, Warrier S, Wang LZ, Kumar AP, Ahn KS, Sethi G, et al: Role of novel histone modifications in cancer. Oncotarget 9: 11414-11426, 2017.

62. Sachdeva M, Dodd RD, Huang Z, Grenier C, Ma Y, Lev DC, Cardona DM, Murphy SK and Kirsch DG: Epigenetic silencing of Kruppel like factor-3 increases expression of pro-metastatic miR-182. Cancer Lett 369: 202-211, 2015.

63. Kanherkar RR, Bhatia-Dey N and Csoka AB: Epigenetics across the human lifespan. Front Cell Dev Biol 2: 49, 2014.

64. Sadikovic B, Yoshimoto M, Al-Romaih K, Maire G, Zielenska M and Squire JA: In vitro analysis of integrated global high-resolution DNA methylation profiling with genomic imbalance and gene expression in osteosarcoma. PLoS One 3: e2834, 2008.

65. Kresse SH,RydbeckH,Skårn M,Namløs HM,Barragan-Polania AH, Cleton-Jansen AM, Serra M, Liest $\varnothing 1$ K, Hogendoorn PC, Hovig E, et al: Integrative analysis reveals relationships of genetic and epigenetic alterations in osteosarcoma. PLoS ONE 7: e48262, 2012.

66. Rosenblum JM, Wijetunga NA, Fazzari MJ, Krailo M, Barkauskas DA, Gorlick R and Greally JM: Predictive properties of DNA methylation patterns in primary tumor samples for osteosarcoma relapse status. Epigenetics 10: 31-39, 2015.

67. Varshney J, Scott MC, Largaespada DA and Subramanian S: Understanding the osteosarcoma pathobiology-a comparative oncology approach. Vet Sci 3: pii: E3, 2016.

68. Jeziorska DM, Murray RJS, De Gobbi M, Gaentzsch R, Garrick D, Ayyub H, Chen T, Li E, Telenius J, Lynch M, et al: DNA methylation of intragenic $\mathrm{CpG}$ islands depends on their transcriptional activity during differentiation and disease. Proc Natl Acad Sci USA 114: E7526-E7535, 2017.

69. Li B and Ye Z: Epigenetic alterations in osteosarcoma: Promising targets. Mol Biol Rep 41: 3303-3315, 2014.

70. Hamidi T, Singh AK and Chen T: Genetic alterations of DNA methylation machinery in human diseases. Epigenomics 7 : $247-265,2015$.

71. Li E and Zhang Y: DNA methylation in mammals. Cold Spring Harb Perspect Biol 6: a019133, 2014

72. Goll MG, Kirpekar F, Maggert KA, Yoder JA, Hsieh CL, Zhang X, Golic KG, Jacobsen SE and Bestor TH: Methylation of tRNA Asp by the DNA methyltransferase homolog Dnmt2. Science 311: 395-398, 2006

73. Pfeifer GP: Defining driver DNA methylation changes in human cancer. Int J Mol Sci 19: pii: E1166, 2018.

74. Al-Romaih K, Sadikovic B, Yoshimoto M, Wang Y, Zielenska M and Squire JA: Decitabine-induced demethylation of 5 ' $\mathrm{CpG}$ island in GADD45A leads to apoptosis in osteosarcoma cells. Neoplasia 10: 471-480, 2008

75. Amente S, Zhang J, Lavadera ML, Lania L, Avvedimento EV and Majello B: Myc and PI3K/AKT signaling cooperatively repress FOXO3a-dependent PUMA and GADD45a gene expression. Nucleic Acids Res 39: 9498-9507, 2011.

76. Zhang H, He J, Li J, Tian D, Gu L and Zhou M: Methylation of RASSF1A gene promoter is regulated by p53 and DAXX. FASEB J 27: 232-242, 2013

77. Song MS, Song SJ, Kim SY, Oh HJ and Lim DS: The tumour suppressor RASSF1A promotes MDM2 self-ubiquitination by disrupting the MDM2-DAXX-HAUSP complex. EMBO J 27: 1863-1874, 2008

78. Guo X, Liu W, Pan Y, Ni P, Ji J, Guo L, Zhang J, Wu J, Jiang J, Chen X, et al: Homeobox gene IRX1 is a tumor suppressor gene in gastric carcinoma. Oncogene 29: 3908-3920, 2010.

79. Moriarity BS, Otto GM, Rahrmann EP, Rathe SK, Wolf NK, Weg MT, Manlove LA, LaRue RS, Temiz NA, Molyneux SD, et al: A sleeping beauty forward genetic screen identifies new genes and pathways driving osteosarcoma development and metastasis. Nat Genet 47: 615-624, 2015.

80. Xu J, Li D, Cai Z, Zhang Y, Huang Y, Su B and Ma R: An integrative analysis of DNA methylation in osteosarcoma. J Bone Oncol 9: 34-40, 2017

81. Li X, Lu H, Fan G, He M, Sun Y, Xu K and Shi F: A novel interplay between HOTAIR and DNA methylation in osteosarcoma cells indicates a new therapeutic strategy. J Cancer Res Clin Oncol 143: 2189-2200, 2017.

82. Wang Q: CpG methylation patterns are associated with gene expression variation in osteosarcoma. Mol Med Rep 16: 901-907, 2017

83. Li Q, Li H, Zhao X, Wang B, Zhang L, Zhang C and Zhang F: DNA methylation mediated downregulation of miR-449c controls osteosarcoma cell cycle progression by directly targeting oncogene c-Myc. Int J Biol Sci 13: 1038-1050, 2017. 
84. Tian W, Li Y, Zhang J, Li J and Gao J: Combined analysis of DNA methylation and gene expression profiles of osteosarcoma identified several prognosis signatures. Gene 650: 7-14, 2018.

85. Bannister AJ and Kouzarides T: Regulation of chromatin by histone modifications. Cell Res 21: 381-395, 2011

86. Li S: Implication of posttranslational histone modifications in nucleotide excision repair. Int J Mol Sci 13: 12461-12486, 2012.

87. Kelly TK, De Carvalho DD and Jones PA: Epigenetic modifications as therapeutic targets. Nat Biotechnol 28: 1069-1078, 2010.

88. Ropero S and Esteller M: The role of histone deacetylases (HDACs) in human cancer. Mol Oncol 1: 19-25, 2007.

89. Black JC, Van Rechem C and Whetstine JR: Histone lysine methylation dynamics: Establishment, regulation, and biological impact. Mol Cell 48: 491-507, 2012.

90. Vaidya H, Rumph C and Katula KS: Inactivation of the WNT5A alternative promoter $\mathrm{B}$ is associated with DNA methylation and histone modification in osteosarcoma cell lines U2OS and SaOS-2. PLoS One 11: e0151392, 2016.

91. He C, Sun J, Liu C, Jiang Y and Hao Y: Elevated H3K27me3 levels sensitize osteosarcoma to cisplatin. Clin Epigenetics 11: 8, 2019.

92.Lawlor ER and Thiele CJ: pigenetic changes in pediatric solid tumors: Promising new targets. Clin Cancer Res 18: 2768-2779, 2012

93. Wu SC and Benavente CA: Chromatin remodeling protein HELLS is upregulated by inactivation of the RB-E2F pathway and is nonessential for osteosarcoma tumorigenesis. Oncotarget 9: 32580-32592, 2018.

94. Nie JH, Li TX, Zhang XQ and Liu J: Roles of non-coding RNAs in normal human brain development, brain tumor, and neuropsychiatric disorders. Noncoding RNA 5: pii: E36, 2019.

95. Fernandes JCR, Acuña SM, Aoki JI, Floeter-Winter LM and Muxel SM: Long non-coding RNAs in the regulation of gene expression: Physiology and disease. Noncoding RNA 5: pii: E17, 2019.

96. Calin GA: The noncoding RNA revolution-three decades and still going strong! Mol Oncol 13: 3, 2019.

97. Patil VS, Zhou R and Rana TM: Gene regulation by non-coding RNAs. Crit Rev Biochem Mol Biol 49: 16-32, 2014.

98. Mens MMJ and Ghanbari M: Cell cycle regulation of stem cells by microRNAs. Stem Cell Rev Rep 14: 309-322, 2018

99. O'Brien J, Hayder H, Zayed Y and Peng C: Overview of microRNA biogenesis, mechanisms of actions, and circulation. Front Endocrinol (Lausanne) 9: 402, 2018

100. Li Y, Zeng C, Tu M, Jiang W, Dai Z, Hu Y, Deng Z and Xiao W: MicroRNA-200b acts as a tumor suppressor in osteosarcoma via targeting ZEB1. Onco Targets Ther 9: 3101-3111, 2016.

101. Jiang R, Zhang C, Liu G, Gu R and Wu H: MicroRNA-101 inhibits proliferation, migration and invasion in osteosarcoma cells by targeting ROCK1. Am J Cancer Res 7: 88-97, 2017.

102. Xu H, Liu X and Zhao J: Down-regulation of miR-3928 promoted osteosarcoma growth. Cell Physiol Biochem 33 1547-1556, 2014.

103. Huang G, Nishimoto K, Zhou Z, Hughes D and Kleinerman ES miR-20a encoded by the miR-17-92 cluster increases the metastatic potential of osteosarcoma cells by regulating Fas expression. Cancer Res 72: 908-916, 2012.

104. Xu H, Liu X, Zhou J, Chen X and Zhao J: miR-574-3p acts as a tumor promoter in osteosarcoma by targeting SMAD4 signaling pathway. Oncol Lett 12: 5247-5253, 2016.

105. Xiao Q, Huang L, Zhang Z, Chen X, Luo J, Zhang Z, Chen S, Shu Y, Han Z and Cao K: Overexpression of miR-140 inhibits proliferation of osteosarcoma cells via suppression of histone deacetylase 4. Oncol Res 25: 267-275, 2017.

106. Luo Z, Liu M, Zhang H and Xia Y: Association of circulating miR-125b and survival in patients with osteosarcoma-A single center experience. J Bone Oncol 5: 167-172, 2016.

107. Qu Y, Pan S, Kang M, Dong R and Zhao J: MicroRNA-150 functions as a tumor suppressor in osteosarcoma by targeting IGF2BP1. Tumour Biol 37: 5275-5284, 2016.

108. Ali A, Hu L, Qian A, Chen C and Yang T: Long noncoding RNAs and human osteosarcoma. J Stem Cell Res Ther 8: 3, 2018

109. Guo W, Jiang H, Li H, Li F, Yu Q, Liu Y, Jiang W and Zhang M: LncRNA-SRA1 suppresses osteosarcoma cell proliferation while promoting cell apoptosis. Technol Cancer Res Treat 18: $1-11,2019$.

110. Deng R, Zhang J and Chen J: IncRNA SNHG1 negatively regulates miRNA-101-3p to enhance the expression of ROCK1 and promote cell proliferation, migration and invasion in osteosarcoma. Int J Mol Med 43: 1157-1166, 2019.
111. Zhou Y, Yin L, Li H, Liu LH and Xiao T: The LncRNA LINC00963 facilitates osteosarcoma proliferation and invasion by suppressing miR-204-3p/FN1 axis. Cancer Biol Ther 20: 1141-1148, 2019.

112. Chen Y, Huang W, Sun W, Zheng B, Wang C, Luo Z, Wang J and Yan W: LncRNA MALAT1 promotes cancer metastasis in osteosarcoma via activation of the PI3K-Akt signaling pathway. Cell Physiol Biochem 51: 1313-1326, 2018.

113. Gu W, Zhang E, Song L, Tu L, Wang Z, Tian F, Aikenmu K, Chu $G$ and Zhao J: Long noncoding RNA HOXD-AS1 aggravates osteosarcoma carcinogenesis through epigenetically inhibiting p57 via EZH2. Biomed Pharmacother 106: 890-895, 2018.

114. Yu X, Hu L, Li S, Shen J, Wang D, Xu R and Yang H: Long non-coding RNA Taurine upregulated gene 1 promotes osteosarcoma cell metastasis by mediating HIF-1 $\alpha$ via miR-143-5p. Cell Death Dis 10: 280, 2019.

115. Kun-Peng Z, Xiao-Long M and Chun-Lin Z: Overexpressed circPVT1, a potential new circular RNA biomarker, contributes to doxorubicin and cisplatin resistance of osteosarcoma cells by regulating ABCB1. Int J Biol Sci 14: 321-330, 2018.

116. Vo JN, Cieslik M, Zhang Y, Shukla S, Xiao L, Zhang Y, Wu YM, Dhanasekaran SM, Engelke CG, Cao X, et al: The landscape of circular RNA in cancer. Cell 176: 869-881.e13, 2019.

117. Wang C, Ren M, Zhao X, Wang A and Wang J: Emerging roles of circular RNAs in osteosarcoma. Med Sci Monit 24: 7043-7050, 2018.

118. Wu Y, Xie Z, Chen J, Chen J, Ni W, Ma Y, Huang K, Wang G, Wang J, Ma J, et al: Circular RNA circTADA2A promotes osteosarcoma progression and metastasis by sponging miR-203a-3p and regulating CREB3 expression. Mol Cancer 18: 73, 2019.

119. Liu W, Zhang J, Zou C, Xie X, Wang Y, Wang B, Zhao Z, Tu J, Wang $\mathrm{X}, \mathrm{Li} \mathrm{H}$, et al: Microarray expression profile and functional analysis of circular RNAs in osteosarcoma. Cell Physiol Biochem 43: 969-985, 2017.

120. Goldszmid RS and Trinchieri G: The price of immunity. Nat Immunol 13: 932-938, 2012

121. Turtle CJ, Hudecek M, Jensen MC and Riddell SR: Engineered T cells for anti-cancer therapy. Curr Opin Immunol 24: 633-639, 2012.

122. Tu B, Du L, Fan QM, Tang Z and Tang TT: STAT3 activation by IL-6 from mesenchymal stem cells promotes the proliferation and metastasis of osteosarcoma. Cancer Lett 325: 80-88, 2012.

123. Wu Z, Yang W, Liu J and Zhang F: Interleukin-6 upregulates SOX18 expression in osteosarcoma. Onco Targets Ther 10: 5329-5336, 2017.

124. Tzeng HE, Tsai CH, Chang ZL, Su CM, Wang SW, Hwang WL and Tang CH: Interleukin- 6 induces vascular endothelial growth factor expression and promotes angiogenesis through apoptosis signal-regulating kinase 1 in human osteosarcoma. Biochem Pharmacol 85: 531-540, 2013.

125. Wu Q, Zhou X, Huang D, Ji Y and Kang F: IL-6 enhances osteocyte-mediated osteoclastogenesis by promoting JAK2 and RANKL activity in vitro. Cell Physiol Biochem 41: 1360-1369, 2017.

126. Gross AC, Cam H, Phelps DA, Saraf AJ, Bid HK, Cam M, London CA, Winget SA, Arnold MA, Brandolini L, et al: IL-6 and CXCL8 mediate osteosarcoma-lung interactions critical to metastasis. JCI Insight 3: pii: 99791, 2018.

127. Zhang $\mathrm{H}$, Wu H, Zheng J, Yu P, Xu L, Jiang P, Gao J, Wang H and Zhang Y: Transforming growth factor $\beta 1$ signal is crucial for dedifferentiation of cancer cells to cancer stem cells in osteosarcoma. Stem Cells 31: 433-446, 2013.

128. Lamora A, Talbot J, Mullard M, Brounais-Le Royer B, Redini F and Verrecchia F: TGF- $\beta$ signaling in bone remodeling and osteosarcoma progression. J Clin Med 5: pii: E96, 2016.

129. Li F, Li S and Cheng T: TGF- $\beta 1$ promotes osteosarcoma cell migration and invasion through the miR-143-versican pathway. Cell Physiol Biochem 34: 2169-2179, 2014.

130. Verrecchia $\mathrm{F}$ and Rédini F: Transforming growth factor- $\beta$ signaling plays a pivotal role in the interplay between osteosarcoma cells and their microenvironment. Front Oncol 8: 133, 2018.

131. Lebrun JJ: The dual role of TGF $\beta$ in human cancer: From tumor suppression to cancer metastasis. ISRN Mol Biol 2012: 381428, 2012.

132. Cantelli G, Crosas-Molist E, Georgouli M and Sanz-Moreno V: TGFB-induced transcription in cancer. Semin Cancer Biol 42: 60-69, 2017 
133. Baglio SR, Lagerweij T, Pérez-Lanzón M, Ho XD, Léveillé N, Melo SA, Cleton-Jansen AM, Jordanova ES, Roncuzzi L, Greco M, et al: Blocking tumor-educated MSC paracrine activity halts osteosarcoma progression. Clin Cancer Res 23: 3721-3733, 2017.

134. Wang T, Wang D, Zhang L, Yang P, Wang J, Liu Q, Yan F and Lin F: The TGF $\beta$-miR-499a-SHKBP1 pathway induces resistance to EGFR inhibitors in osteosarcoma cancer stem cell-like cells. J Exp Clin Cancer Res 38: 226, 2019.

135. Kato H, Wakabayashi H, Naito Y, Kato S, Nakagawa T, Matsumine A and Sudo A: Anti-tumor necrosis factor therapy inhibits lung metastasis in an osteosarcoma cell line. Oncology 88: 139-146, 2015.

136. Ishikawa T, Shimizu T, Ueki A, Yamaguchi SI, Onishi N, Sugihara E, Kuninaka S, Miyamoto T, Morioka $\mathrm{H}$, Nakayama R, et al: Twist2 functions as a tumor suppressor in murine osteosarcoma cells. Cancer Sci 104: 880-888, 2013.

137. Lin H, Lee E, Hestir K, Leo C, Huang M, Bosch E, Halenbeck R, Wu G, Zhou A, Behrens D, et al: Discovery of a cytokine and its receptor by functional screening of the extracellular proteome. Science 320: 807-811, 2008.

138. Wang Y, Szretter KJ, Vermi W, Gilfillan S, Rossini C, Cella M, Barrow AD, Diamond MS and Colonna M: IL-34 is a tissue-restricted ligand of CSF1R required for the development of Langerhans cells and microglia. Nat Immunol 13: 753-760, 2012.
139. Foucher ED, Blanchard S, Preisser L, Garo E, Ifrah N, Guardiola P, Delneste Y and Jeannin P: IL-34 induces the differentiation of human monocytes into immunosuppressive macrophages-Antagonistic effects of GM-CSF and IFN $\gamma$. PLoS One 8: e56045, 2013.

140. Ségaliny AI, Mohamadi A, Dizier B, Lokajczyk A, Brion R, Lanel R, Amiaud J, Charrier C, Boisson-Vidal C and Heymann D: Interleukin-34 promotes tumor progression and metastatic process in osteosarcoma through induction of angiogenesis and macrophage recruitment. Int J Cancer 137: 73-85, 2015.

141. Honorati MC, Cattini L and Facchini A: Possible prognostic role of IL-17R in osteosarcoma. J Cancer Res Clin Oncol 133: 1017-1021, 2007.

142. Wang M, Wang L, Ren T, Xu L and Wen Z: IL-17A/IL-17RA interaction promoted metastasis of osteosarcoma cells. Cancer Biol Ther 14: 155-163, 2013.

This work is licensed under a Creative Commons Attribution-NonCommercial-NoDerivatives 4.0 International (CC BY-NC-ND 4.0) License. 